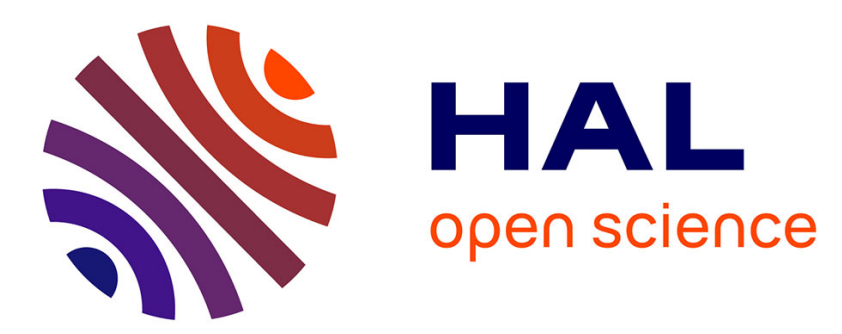

\title{
Imaging an acoustic waveguide from surface data in the time domain
}

\author{
Vahan Baronian, Laurent Bourgeois, Arnaud Recoquillay
}

\section{To cite this version:}

Vahan Baronian, Laurent Bourgeois, Arnaud Recoquillay. Imaging an acoustic waveguide from surface data in the time domain. Wave Motion, 2016, 66, pp.68 - 87. 10.1016/j.wavemoti.2016.05.006 . hal01379890

\section{HAL Id: hal-01379890 \\ https://inria.hal.science/hal-01379890}

Submitted on 12 Oct 2016

HAL is a multi-disciplinary open access archive for the deposit and dissemination of scientific research documents, whether they are published or not. The documents may come from teaching and research institutions in France or abroad, or from public or private research centers.
L'archive ouverte pluridisciplinaire $\mathbf{H A L}$, est destinée au dépôt et à la diffusion de documents scientifiques de niveau recherche, publiés ou non, émanant des établissements d'enseignement et de recherche français ou étrangers, des laboratoires publics ou privés. 


\title{
Imaging an acoustic waveguide from surface data in the time domain
}

\author{
Vahan Baronian $^{\mathrm{a}}$, Laurent Bourgeois ${ }^{\mathrm{b}, *}$, Arnaud Recoquillay ${ }^{\mathrm{a}, \mathrm{b}}$ \\ ${ }^{a} C E A$, LIST, Gif-sur-Yvette, France \\ ${ }^{b}$ Laboratoire POEMS, UMR ENSTA/CNRS/INRIA, ENSTA ParisTech, 828 Boulevard des \\ Maréchaux, 91120 Palaiseau, France
}

\begin{abstract}
This paper deals with an inverse scattering problem in an acoustic waveguide. The data consist of time domain signals given by sources and receivers located on the boundary of the waveguide. After transforming the data to the frequency domain, the obstacle is then recovered by using a modal formulation of the Linear Sampling Method. The impact of many parameters are analyzed, such as the numbers of sources/receivers and the distance between them, the time shape of the incident wave and the number and the values of the frequencies that are used. Some numerical experiments illustrate such analysis.
\end{abstract}

Keywords: Acoustic waveguide, Inverse scattering problem, Linear Sampling Method, Time domain, Surface data.

\section{Introduction}

This paper considers the inverse problem of identifying an obstacle in an acoustic waveguide by applying some sources on some part of the boundary of the waveguide and by measuring the corresponding scattered fields on the

5 same part of the boundary. Both the sources and the receivers are supposed to be far away from the obstacle and the data are given in the time domain. Our contribution can be considered as an improvement of [1] in the sense that the data are more realistic. Firstly, in accordance with the experiment of Non Destructive Evaluation, the sources and receivers are located at the boundary of the waveguide instead of inside the waveguide. Secondly, we handle data in the time domain instead of data in the frequency domain, which is also closer to a realistic experiment. Quite often in experimental setups, the sources and receivers are piezoelectric transducers or laser vibrometers that are indeed placed on the surface of the structure to image. The transducers impose some 15 localized distributed sources while the measurements with lasers are allowed to

\footnotetext{
* Corresponding author

Email address: laurent.bourgeois@ensta.fr (Laurent Bourgeois)
} 


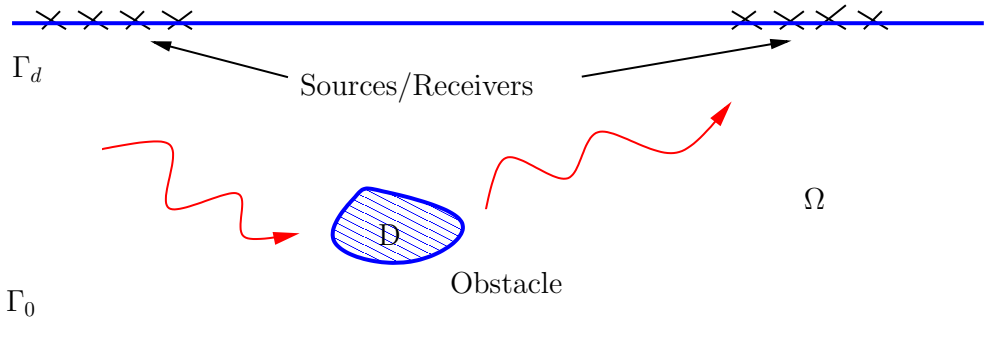

Figure 1: Configuration of the inverse problem

be point values. Among the huge literature on experimental validation of NDE, the reader can for example refer to [2, 3, 4, which concern ultrasonic inspection of metallic plates.

We consider a $2 \mathrm{D}$ waveguide $W=\mathbb{R} \times(0, d)$, where $d>0$ is the height of the waveguide, a generic point in $W$ having coordinates $\left(x_{1}, x_{2}\right)$. The boundary of $W$ is denoted $\Gamma=\Gamma_{0} \cup \Gamma_{d}$, where $\Gamma_{0}$ (resp. $\Gamma_{d}$ ) corresponds to $x_{2}=0$ (resp. $x_{2}=d$ ), and the outward unit normal is denoted $\nu$. Let us consider a compactly supported function $f$ of $x_{1}$ and a compactly supported function $\chi$ of time $t$, with $\operatorname{supp}(\chi) \in[0,+\infty)$. For some impenetrable obstacle $D$ such that $\bar{D} \subset W$, let us

25 denote $\Omega=W \backslash \bar{D}$ and assume that $\Omega$ is connected. For some uniform sound speed $c>0$, we consider the solution $v$ in $\Omega \times(0,+\infty)$ to the scattering problem with Neumann data

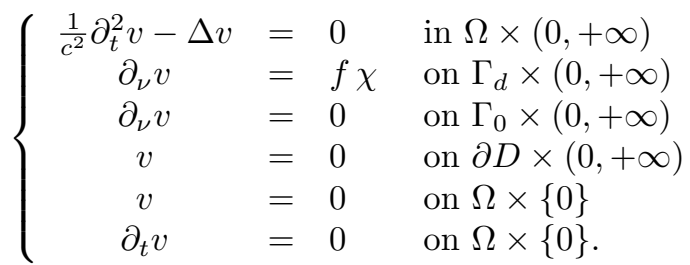

The function of time $\chi$ being fixed, the function of space $f$ is alternatively chosen as $f\left(x_{1}\right)=g\left(x_{1}-x_{1}^{s \pm}\right)$, where $g$ is a compactly supported and even function sо while $x_{1}^{s \pm}$ are the source locations. The solution $v$ is measured on $\Gamma_{d}$ at several points $x_{1}=x_{1}^{r \pm}$ for all time $t \in(0,+\infty)$. We assume that the position of sources and receivers are given by

$$
x_{1}^{m \pm}= \pm(R+m \delta), \quad m=0, \cdots, M-1,
$$

where $R>0$ is such that the obstacle $D$ is a priori located between the transverse sections of coordinates $x_{1}=-R$ and $x_{1}=R$ and $\delta>0$. Well-posedness of 35 problem (1) for reasonable data $(f, \chi)$ is well-known (see for example [5]). The objective is to identify the obstacle $D$ from all time signals obtained for the $2 M$ sources and the $2 M$ receivers. The configuration of our inverse problem is illustrated in figure 1.

Our strategy is based on the transformation of the data to the frequency domain and then the use of the Linear Sampling Method (LSM) in a modal 
formulation at several fixed frequencies. The LSM was first introduced in [6] in free space and has been successfully applied in many situations (see for example [7]). The application of sampling methods to acoustic waveguides in the frequency regime goes back to $[8$ and we mention [9, 1, 10, as significant contri45 butions to the field. In particular, in [1], the specific geometry of the waveguide is exploited in order to derive a well-adapted and efficient modal formulation of the LSM. In [11 the sampling methods are adapted to image cracks instead of obstacles located inside the waveguide, while the case of the elastic instead of acoustic waveguide is analyzed in [12, 13. It should be noted that a "time

50 domain" version of the Linear Sampling Method was introduced in [14 in free space. A first attempt to adapt such method to the case of acoustic waveguide has very recently been proposed in [15. In contrast with our own contribution, the authors of [15] handle data directly in the time domain without transforming them to the frequency domain, which is a fundamental difference. It is also worth noting that in 15 the sources and receivers are both located inside the waveguide.

Our paper is organized as follows. In section 2 we summarize the modal approach presented in [1] in the frequency domain. Such approach can be viewed as an ideal inverse problem in which point sources are located on two transverse 60 sections on both sides of the obstacle and the corresponding scattered fields are measured on these two transverse sections. It is shown in [1] that these data exactly amount to the infinite scattering matrix associated with all the guided modes (propagating and evanescent) and that in the far field approximation (the obstacle is far away from the transverse sections), the data amount to the 65 scattering matrix associated with the propagating guided modes only. In section 3 , which is again limited to the frequency domain, we consider Neumann sources centered at points of the boundary of the waveguide and receivers located at the same points. The main result is that the scattering matrix mentioned above can be recovered from these surface data up to the inversion of two ill-conditioned 70 matrices. It is shown that the number of sources and receivers, that is $2 M$, as well as the distance $\delta$ between them, are two fundamental parameters that are analyzed in order to optimize the condition number of such matrices. Data in the time domain are then considered in section 4 , where the procedure to come back to the previous time harmonic situation is described. In contrast with the 75 case of free space, a fundamental feature of wave propagation in waveguides is the fact that, due to the physical presence of the boundaries, the scattered fields decrease very slowly as a function of the time variable. It is shown that the choice of the time function $\chi$ in problem (1) is critical in order to obtain a scattered field that rapidly vanishes for a large time. This is achieved by ${ }_{80}$ choosing some $\chi$ the Fourier transform of which avoids the cut-off frequencies of the waveguide. The section 5 is dedicated to some numerical experiments, in which we in particular analyze the impact of the number and the values of the frequencies that are used. Lastly, for the sake of completeness the appendix A presents a few results concerning the condition number of Vandermonde matrices 85 with unit complex entries, while the appendix B establishes the expression of the fundamental solution of the waveguide for a Dirac located on its boundary, 
which are two key points of section 3 .

\section{The modal approach of the Linear Sampling Method}

We first introduce what we could consider as an ideal inverse problem and

90 we have presented in the introduction. The reason why we introduce such ideal inverse problem is that it enables us to easily justify the Linear Sampling Method. More precisely, it consists of a modal approach of the LSM in the frequency domain and was first introduced in 11. We now recall the main results of [1. Let $k>0$ be a wave number and $u^{i}$ an incident field, the corresponding scattered field $u^{s}$ is defined as the solution of the scattering problem

$$
\left\{\begin{array}{ccc}
\left(\Delta+k^{2}\right) u^{s}=0 & \text { in } & W \backslash \bar{D} \\
\partial_{\nu} u^{s}=0 & \text { on } & \Gamma \\
u^{s}=-u^{i} & \text { on } & \partial D \\
(R C), & &
\end{array}\right.
$$

where $(R C)$ is a radiation condition that governs the behavior of the solution $u^{s}$ at infinity. Such condition, which is not necessary to specify here, can be expressed with the help of the Dirichlet-to-Neumann operators on some transverse sections on both side of the defect $D$ (see [1]) or with the help of the propagating guided modes (see [16]). It is well known that for sufficiently smooth $D$ and $u^{i}$ such problem is well-posed except for at most a countable set of wave numbers $k$. Let us now consider $\hat{\Sigma}=\Sigma_{-R} \cup \Sigma_{R}$, where $\Sigma_{s}$ is the transverse section corresponding to $x_{1}=s$, such that $D$ is a priori strictly surrounded by $\Sigma_{-R}$ and $\Sigma_{R}$. For $y \in \hat{\Sigma}$, let us denote $u^{s}(\cdot, y)$ the solution to problem (3) associated with $u^{i}=G(\cdot, y)$, where $G(\cdot, y)$ is the fundamental solution of the waveguide, that is the solution to problem

$$
\left\{\begin{array}{ccc}
-\left(\Delta+k^{2}\right) G(\cdot, y)=\delta_{y} & \text { in } & W \\
\partial_{\nu} G(\cdot, y)=0 & \text { on } & \Gamma \\
(R C) & &
\end{array}\right.
$$

We measure $u^{s}(x, y)$ for all $x \in \hat{\Sigma}$, so that the data of the ideal inverse problem are the trace on $\hat{\Sigma}$ of the scattered fields associated with all point sources located ${ }_{110}$ on $\hat{\Sigma}$. The configuration of such inverse problem is illustrated in figure 2 The Linear Sampling Method relies on the near field operator $\mathcal{N}: L^{2}(\hat{\Sigma}) \rightarrow L^{2}(\hat{\Sigma})$ such that for $h \in L^{2}(\hat{\Sigma})$,

$$
(\mathcal{N} h)(x)=\int_{\hat{\Sigma}} u^{s}(x, y) h(y) d s(y), \quad x \in \hat{\Sigma} .
$$

The following theorem is proved in [1, 17]:

Theorem Except for at most a countable set of $k$, if for some $z \in W$ we 


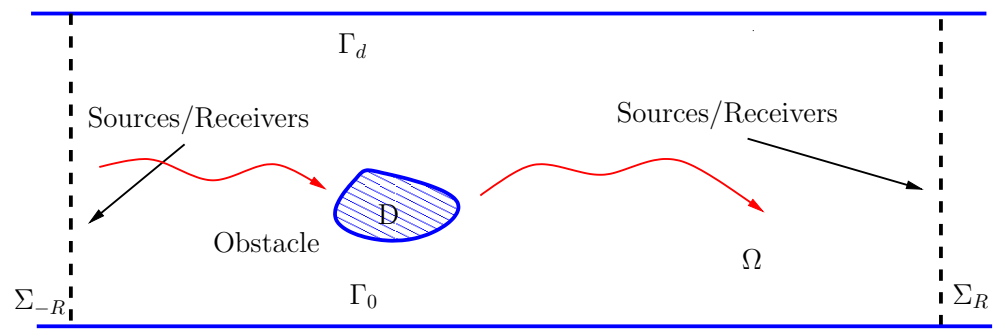

Figure 2: Configuration of the ideal inverse problem

The converse statement is false in general, but the following weaker result is proved in 11, 17]: except for a countable set of frequencies $k$, if $z \in D$, then for all $\varepsilon>0$ there exists a solution $h_{\varepsilon}(\cdot, z) \in L^{2}(\hat{\Sigma})$ of the inequality $\left\|\mathcal{N} h_{\varepsilon}(\cdot, z)-G(\cdot, z)\right\|_{L^{2}(\hat{\Sigma})} \leq \varepsilon$ such that for a given fixed $\varepsilon$, the function $h_{\varepsilon}(\cdot, z)$ satisfies $\lim _{z \rightarrow \partial \mathcal{D}}\left\|h_{\varepsilon}(\cdot, z)\right\|_{L^{2}(\hat{\Sigma})}=+\infty$.

Then a practical method to identify $D$ from the operator $\mathcal{N}$ consists, for all $z$ in some sampling grid, to solve in $L^{2}(\hat{\Sigma})$ the equation

$$
\mathcal{N} h=\left.G(\cdot, z)\right|_{\hat{\Sigma}}
$$

and then to plot the function $\psi(z)=1 /\|h(z)\|_{L^{2}(\hat{\Sigma})}$, which from the above theorem turns out to be an indicator function of the defect.

Our modal approach consists in taking advantage of the specific geometry of the waveguide. Classically, the solutions to the Neumann eigenvalue problem of the negative 1D Laplacian in some transverse section $\Sigma$ are $\lambda_{n}=n^{2} \pi^{2} / d^{2}$ for $n \in \mathbb{N}$ and the corresponding eigenvectors $\theta_{n}$ form an orthonormal basis of $L^{2}(\Sigma)$. The $\theta_{n}$ are given by

$$
\left\{\begin{array}{c}
\theta_{0}\left(x_{2}\right)=\sqrt{\frac{1}{d}} \\
\theta_{n}\left(x_{2}\right)=\sqrt{\frac{2}{d}} \cos \left(\frac{n \pi}{d} x_{2}\right) \quad(n \geq 1) .
\end{array}\right.
$$

The solutions to the problem

$$
\left\{\begin{array}{ccc}
\left(\Delta+k^{2}\right) u=0 & \text { in } & W \\
\partial_{\nu} u=0 & \text { on } & \Gamma
\end{array}\right.
$$

are then the linear combinations of the so-called guided modes, defined for $n \in \mathbb{N}$ by

$$
u_{n}^{ \pm}\left(x_{1}, x_{2}\right)=\theta_{n}\left(x_{2}\right) e^{ \pm i \beta_{n} x_{1}}, \quad \beta_{n}=\sqrt{k^{2}-\lambda_{n}}, \quad \operatorname{Re} \beta_{n}, \operatorname{Im} \beta_{n} \geq 0 .
$$

If in addition we assume that $k$ is such that $\beta_{n} \neq 0$ for all $n \geq 0$, there is a finite number $N$ such that the $N$ first $\beta_{n}$ have $\operatorname{Re} \beta_{n}>0$, the remainder of the ${ }_{135} \beta_{n}$ having $\operatorname{Im} \beta_{n}>0$. Consequently, for $n=0, \cdots, N-1$ the guided modes $u_{n}^{+}$ (respectively $u_{n}^{-}$) are propagating from the left to the right of the waveguide 
(respectively from the right to the left), while for $n \geq N$ the guided modes $u_{n}^{+}$ (respectively $u_{n}^{-}$) are decaying exponentially from the left to the right of the waveguide (respectively from the right to the left). The modal version of the Linear Sampling Method is obtained by projecting the relationship (5) along the complete basis of $L^{2}\left(\Sigma_{ \pm R}\right)$ formed by the functions $\theta_{n}$. We denote by $u_{n}^{s \pm}$ the solution for $n \in \mathbb{N}$ to problem (3) with $u^{i}=-u_{n}^{ \pm}$. by

Since the fundamental solution of the waveguide $G$ is given, for all $x, y \in W$,

$$
G(x, y)=-\sum_{n \in \mathbb{N}} \frac{e^{i \beta_{n}\left|x_{1}-y_{1}\right|}}{2 i \beta_{n}} \theta_{n}\left(x_{2}\right) \theta_{n}\left(y_{2}\right),
$$

by denoting $h=\left(h^{-}, h^{+}\right) \in L^{2}\left(\Sigma_{-R}\right) \times L^{2}\left(\Sigma_{R}\right)$, with

$$
h^{-}=\sum_{m \in \mathbb{N}} h_{m}^{-} \theta_{m}, \quad h^{+}=\sum_{m \in \mathbb{N}} h_{m}^{+} \theta_{m}
$$

and

$$
\left.u_{n}^{s+}\right|_{\Sigma_{ \pm R}}=\sum_{m \in \mathbb{N}} S_{m n}^{+ \pm} \theta_{m},\left.\quad u_{n}^{s-}\right|_{\Sigma_{ \pm R}}=\sum_{m \in \mathbb{N}} S_{m n}^{- \pm} \theta_{m}
$$

it is proved in [1] that the equation (5] is equivalent to the infinite system

$$
\forall m \in \mathbb{N}, \quad\left\{\begin{array}{l}
\sum_{n \in \mathbb{N}} \frac{e^{i \beta_{n} R}}{2 i \beta_{n}}\left(S_{m n}^{+-} h_{n}^{-}+S_{m n}^{--} h_{n}^{+}\right)=\frac{e^{i \beta_{m}\left(R+z_{1}\right)}}{2 i \beta_{m}} \theta_{m}\left(z_{2}\right) \\
\sum_{n \in \mathbb{N}} \frac{e^{i \beta_{n} R}}{2 i \beta_{n}}\left(S_{m n}^{++} h_{n}^{-}+S_{m n}^{-+} h_{n}^{+}\right)=\frac{e^{i \beta_{m}\left(R-z_{1}\right)}}{2 i \beta_{m}} \theta_{m}\left(z_{2}\right) .
\end{array}\right.
$$

This shows that our ideal inverse problem consists equivalently:

- to know the scattered fields on $\hat{\Sigma}$ associated to all point sources on $\hat{\Sigma}$,

- to know the projections on the $\theta_{m}$ functions of the scattered fields on $\hat{\Sigma}$ associated to all guided modes $\left(u_{n}^{ \pm}\right)$for $m, n \in \mathbb{N}$.

We show in [1] that the infinite system (9) is ill-posed since the underlying operator $\mathcal{N}$ to invert is compact. We also show in 1] that a relevant way to regularize this infinite system by discretization consists in limiting the indices $n$ to the first $N$ integers, where $N$ is the number of propagating guided modes. It amounts to restrict the information contained in the scattering data used in the ideal inverse problem to the subpart which propagates at long distance (the evanescent part is not taken into account). It turns out that this restriction is efficient and does not require additional regularization as soon as the number of propagating modes is relatively small, for instance less than 12 as it will be the case in our numerical experiments. We hence define the matrices and vectors

$$
\begin{gathered}
S^{- \pm}=\left(S_{m n}^{- \pm}\right), \quad S^{+ \pm}=\left(S_{m n}^{+ \pm}\right), \quad m, n=0, \cdots N-1 \\
H^{ \pm}=\left(h_{m}^{ \pm}\right), \quad F^{ \pm}=\left(\frac{e^{i \beta_{m}\left(R \mp z_{1}\right)}}{2 i \beta_{m}} \theta_{m}\left(z_{2}\right)\right), \quad m=0, \cdots N-1,
\end{gathered}
$$


while $K$ is the $N \times N$ diagonal matrix formed by the diagonal terms $\left(e^{i \beta_{n} R} / 2 i \beta_{n}\right)$, $n=0, \cdots, N-1$, so that the infinite system (9) becomes the $2 N \times 2 N$ system

$$
\mathcal{U} H=F
$$

\section{Surface data in the frequency domain}

The $2 N \times 2 N$ matrix $\mathcal{S}$ is known as the scattering matrix in the literature while the $2 N \times 2 N$ matrix $\mathcal{U}$ will be the so-called LSM matrix. In what follows it will be convenient to introduce the matrices $U^{- \pm}=S^{- \pm} K, U^{+ \pm}=S^{+ \pm} K$.

\subsection{On the use of convolution}

Let $k>0$ be a wave number and $\phi$ a compactly supported function of $x_{1}$, we consider the solution $u$ to the scattering problem

$$
\left\{\begin{array}{ccc}
\left(\Delta+k^{2}\right) u=0 & \text { in } & W \backslash \bar{D} \\
\partial_{\nu} u=\phi & \text { on } & \Gamma_{d} \\
\partial_{\nu} u=0 & \text { on } & \Gamma_{0} \\
u=0 & \text { on } & \partial D \\
(R C) . & &
\end{array}\right.
$$

Again it is well known that except for a countable set of $k$ the problem 11 is well-posed for sufficiently smooth $\phi$. Let us first consider the particular case $\phi=\delta_{y_{1}}$. It is useful to remark that for any $y_{1} \in \mathbb{R}$ and $y_{2}=d$ (see appendix B), the fundamental solution $G(\cdot, y)$ defined by $(8)$ is the solution to the problem

$$
\left\{\begin{array}{ccc}
\left(\Delta+k^{2}\right) G(\cdot, y)=0 & \text { in } & W \\
\partial_{\nu} G(\cdot, y)=\delta_{y_{1}} & \text { on } & \Gamma_{d} \\
\partial_{\nu} G(\cdot, y)=0 & \text { on } & \Gamma_{0} \\
(R C) . & &
\end{array}\right.
$$

We conclude that for $\phi=\delta_{y_{1}}$, if $u$ is the solution to problem (11) the scattered field $u^{s}=u-G(\cdot, y)$ is the solution to problem (3) with $u^{i}=G(\cdot, y)$. For some general compactly supported function $\phi$, using formula (8) and convolution, the 180 scattered field $u^{s}$ associated with problem (11) is the solution to problem (3) with

$$
u^{i}\left(x_{1}, x_{2}\right)=-\sum_{n \in \mathbb{N}} \frac{1}{2 i \beta_{n}} \theta_{n}\left(x_{2}\right) \theta_{n}(d) \int_{\mathbb{R}} e^{i \beta_{n}\left|x_{1}-y_{1}\right|} \phi\left(y_{1}\right) d y_{1} .
$$




\subsection{The measurement matrix}

From now on, we assume that $\phi$ is one of the functions $g\left(\cdot-x_{1}^{s \pm}\right)$ centered at $x_{1}^{s \pm}= \pm(R+s \delta)$ for a given compactly supported and even function $g$ as defined in the introduction with $s=0, \cdots, M-1$. We assume that $M \geq N$. For $x_{1}^{s-}=-R-s \delta$, from the expression 12 the incident field $u^{i}$ can be rewritten

$$
\begin{gathered}
u^{i}=-\sum_{n \in \mathbb{N}} \frac{1}{2 i \beta_{n}} \theta_{n}(d)\left(\int_{\mathbb{R}} e^{-i \beta_{n} y_{1}} g\left(y_{1}+R+s \delta\right) d y_{1}\right) u_{n}^{+} \\
=-\sum_{n \in \mathbb{N}} \frac{e^{i \beta_{n} R}}{2 i \beta_{n}} \alpha_{n} \theta_{n}(d) e^{i s \beta_{n} \delta} u_{n}^{+}
\end{gathered}
$$

with

$$
\alpha_{n}=\int_{\mathbb{R}} e^{-i \beta_{n} z} g(z) d z .
$$

As a consequence, the corresponding scattered field $u^{s}$ satisfies

$$
u^{s}=-\sum_{n \in \mathbb{N}} \frac{e^{i \beta_{n} R}}{2 i \beta_{n}} \alpha_{n} \theta_{n}(d) e^{i s \beta_{n} \delta} u_{n}^{s+} .
$$

Now we will need the following lemma, which is proved in [1]:

Lemma 1 For all $s$ and $h \in H^{1 / 2}\left(\Sigma_{s}\right)$, the following problem

$$
\left\{\begin{array}{ccc}
\left(\Delta+k^{2}\right) u=0 & \text { in } & (s,+\infty) \times(0, d) \\
\partial_{\nu} u=0 & \text { on } & (s,+\infty) \times(\{0\} \cup\{d\}) \\
u=h & \text { on } & \Sigma_{s} \\
(R C) & &
\end{array}\right.
$$

has a unique solution in $H^{1}((s, t) \times(0, d))$ for all $t>s$, which is given by

$$
u\left(x_{1}, x_{2}\right)=\sum_{n \in \mathbb{N}}\left(h, \theta_{n}\right)_{s} e^{i \beta_{n}\left(x_{1}-s\right)} \theta_{n}\left(x_{2}\right),
$$

where $(\cdot, \cdot)_{s}$ is the $L^{2}\left(\Sigma_{s}\right)$ scalar product.

Similarly, the solution in $(-\infty, s)$ is obtained by replacing $\beta_{n}$ by $-\beta_{n}$ in the above expression of $u$.

First we compute the solution $u^{s}$ on $\Gamma_{d}$ at point $x_{1}^{r+}=R+r \delta$. From the above lemma applied to $u_{n}^{s+}$ we obtain

$$
u_{n}^{s+}\left(x_{1}^{r+}, d\right)=\sum_{m \in \mathbb{N}}\left(u_{n}^{s+}(R, \cdot), \theta_{m}\right)_{R} e^{i r \beta_{m} \delta} \theta_{m}(d)=\sum_{m \in \mathbb{N}} S_{m n}^{++} e^{i r \beta_{m} \delta} \theta_{m}(d) .
$$

By plugging the above expression in 14, we obtain

$$
u^{s}\left(x_{1}^{r+}, d\right)=-\sum_{m \in \mathbb{N}} \sum_{n \in \mathbb{N}} e^{i r \beta_{m} \delta} \theta_{m}(d) \frac{e^{i \beta_{n} R}}{2 i \beta_{n}} S_{m n}^{++} \alpha_{n} \theta_{n}(d) e^{i s \beta_{n} \delta} .
$$


As a conclusion, if we approximate the series by sums on the first $N$ terms, $0, \cdots, M-1$ the scattered field at point $(R+r \delta, d)$ due to the source located at point $(-R-s \delta, d)$ can be approximated by $M_{r s}^{++}$given by

$$
M_{r s}^{++}=-\sum_{m=0}^{N-1} \sum_{n=0}^{N-1} e^{i r \beta_{m} \delta} \theta_{m}(d) \frac{e^{i \beta_{n} R}}{2 i \beta_{n}} S_{m n}^{++} \alpha_{n} \theta_{n}(d) e^{i s \beta_{n} \delta}
$$

Let us denote by $V$ the $M \times N$ Vandermonde matrix given by

$$
V_{m n}=e^{i m \beta_{n} \delta}, \quad m=0, \cdots, M-1, \quad n=0, \cdots, N-1
$$

and $A, T$ the $N \times N$ diagonal matrices formed by the diagonal terms $\alpha_{n}, \theta_{n}(d)$ for $n=0, \cdots, N-1$, respectively. The $M \times M$ matrix $M^{++}$has the short expression

$$
M^{++}=-(V T)\left(S^{++} K\right)(V T A)^{t}=-R U^{++} E^{t},
$$

where $R=V T, E=V T A$, and ${ }^{t}$ means transposition. Secondly we compute the solution $u^{s}$ on $\Gamma_{d}$ at point $x_{1}^{r-}=-(R+r \delta)$. From the above lemma applied to $u_{n}^{s+}$ we obtain

$$
u_{n}^{s+}\left(x_{1}^{r+}, d\right)=\sum_{m \in \mathbb{N}}\left(u_{n}^{s+}(-R, \cdot), \theta_{m}\right)_{-R} e^{i r \beta_{m} \delta} \theta_{m}(d)=\sum_{m \in \mathbb{N}} S_{m n}^{+-} e^{i r \beta_{m} \delta} \theta_{m}(d) .
$$

By proceeding as before, we obtain that for $r, s=0, \cdots, M-1$ the scattered field at point $(-R-r \delta, d)$ due to the source located at point $(-R-s \delta, d)$ can be approximated by $M_{r s}^{+-}$corresponding to the matrix

$$
M^{+-}=-R U^{+-} E^{t} .
$$

Now let us consider the sources located at $x_{1}^{s+}=R+s \delta$, from the expression (12) $u^{i}$ can be rewritten

$$
u^{i}=-\sum_{n \in \mathbb{N}} \frac{1}{2 i \beta_{n}} \theta_{n}(d)\left(\int_{\mathbb{R}} e^{i \beta_{n} y_{1}} g\left(y_{1}-R-s \delta\right) d y_{1}\right) u_{n}^{-}
$$

$$
=-\sum_{n \in \mathbb{N}} \frac{e^{i \beta_{n} R}}{2 i \beta_{n}} \alpha_{n} \theta_{n}(d) e^{i s \beta_{n} \delta} u_{n}^{-} .
$$

Here we have used the fact that since $g$ is an even function,

$$
\int_{\mathbb{R}} e^{i \beta_{n} z} g(z) d z=\int_{\mathbb{R}} e^{-i \beta_{n} z} g(z) d z=\alpha_{n} .
$$

Then the corresponding scattered field satisfies

$$
u^{s}=-\sum_{n \in \mathbb{N}} \frac{e^{i \beta_{n} R}}{2 i \beta_{n}} \alpha_{n} \theta_{n}(d) e^{i s \beta_{n} \delta} u_{n}^{s-},
$$


so that for $r, s=0, \cdots, M-1$ the scattered field at point $(-R-r \delta, d)$ due to the source located at point $(R+s \delta, d)$ can be approximated by $M_{r s}^{--}$corresponding

to the matrix

$$
M^{--}=-R U^{--} E^{t}
$$

while the scattered field at point $(R+r \delta, d)$ due to the source located at point $(R+s \delta, d)$ can be approximated by $M_{r s}^{-+}$corresponding to the matrix

$$
M^{-+}=-R U^{-+} E^{t} .
$$

By gathering the above results we obtain that

$$
\mathcal{M}=-\mathcal{R} \mathcal{U} \mathcal{E}^{t}
$$

with

$$
\mathcal{M}=\left(\begin{array}{ll}
M^{+-} & M^{--} \\
M^{++} & M^{-+}
\end{array}\right), \quad \mathcal{R}=\left(\begin{array}{cc}
R & 0 \\
0 & R
\end{array}\right), \quad \mathcal{E}=\left(\begin{array}{cc}
E & 0 \\
0 & E
\end{array}\right) .
$$

${ }_{225}$ Our strategy is now to recover the $2 N \times 2 N$ LSM matrix $\mathcal{U}$ from the $2 M \times 2 M$ measurement matrix $\mathcal{M}$ by solving the system 16 . This enables us then to use the LSM formulation 10 for all $z$ describing some sampling grid in order to image the defect within the waveguide. Solving the system (16) amounts to "inverting" the $2 M \times 2 N$ emission and reception matrices $\mathcal{E}$ and $\mathcal{R}$. In practice we separately solve the four systems

$$
M=-R U E^{t},
$$

where $M$ is any matrix $M=M^{++}, M^{+-}, M^{--}, M^{-+}$and $U$ is the corresponding matrix $U=U^{++}, U^{+-}, U^{--}, U^{-+}$. Recalling that $R=V T$ and $E=V T A$, it is clear that the solvability of the above systems depends on the invertibility of the square matrices $T, A$ and $V^{*} V$, where $V^{*}$ denotes the adjoint matrix of $V$. If these three matrices are invertible, then a straightforward algebraic manipulation shows that $U$ is explicitly given from $M$ by formula

$$
U=-T^{-1}\left(V^{*} V\right)^{-1}\left(V^{*} M V^{* t}\right)\left(V^{*} V\right)^{-t} T^{-1} A^{-1},
$$

where $\cdot^{-t}$ means the transposition composed with inversion (or vice versa). From (6) the diagonal matrix $T$ is invertible since none of the $\theta_{n}(d)$ vanishes. Similarly, from 13 it is easy to choose the function $g$ such that none of the $\alpha_{n}$ vanishes, which implies that $A$ is also invertible. We discuss the invertibility of $V^{*} V$ in the next section.

Remark : we note that for $g=\delta$ we have $\alpha_{n}=1$ for all $n=0, \cdots, N-1$, that is $A$ is the identity matrix, so that in this special case we have $E=R$ and $\mathcal{E}=\mathcal{R}$. It should be noted that in order to simplify the presentation 245 we have assumed that the locations of sources and receivers are the same and that the function $g$ is even. This is clearly not a restriction. But without such assumptions, the matrices $\mathcal{E}$ and $\mathcal{R}$ would be slightly more complicated. Eventually, we could adapt our method to the case when measurements are not pointwise values of the scattered fields but integrals over a small space interval. 


\subsection{Optimizing sources and receivers}

The rectangular Vandermonde matrix $V$ given by 15 depends on the $\beta_{n}$ defined by (7) but also on the number $M$ of sources and receivers and on the smallest distance $\delta$ between two sources or two receivers. Contrary to the $\beta_{n}$, which depend on the geometry of the waveguide, the parameters $M$ and $\delta$ shall LSM matrix $U$ from the measurement matrix $M$ by (17). It is recalled in the appendix that for a Vandermonde matrix with entries on the unit circle, that is

$$
V_{m n}=e^{2 \pi i m f_{n}}, \quad m=0, \cdots, M-1, \quad n=0, \cdots, N-1,
$$

for real numbers $f_{n}$ and $M \geq N$, the matrix $V^{*} V$ is invertible if for all $n^{\prime} \neq n$, $f_{n^{\prime}}-f_{n}$ is not an integer. Here

$$
f_{n}=\frac{1}{2 \pi} \beta_{n} \delta=\sqrt{1-n^{2} \frac{\lambda^{2}}{4 d^{2}}} \frac{\delta}{\lambda}, \quad n=0, \cdots, N-1,
$$

by introducing the wavelength $\lambda=2 \pi / k$ corresponding to wave number $k$. Clearly, we have $\left|f_{n^{\prime}}-f_{n}\right|<\delta / \lambda$ for all $n$ and $n^{\prime}$, so that the condition

$$
\delta \leq \lambda
$$

is sufficient to ensure the invertibility of $V^{*} V$.

From now on we assume that (19) is fulfilled and in view of 17 we wish to optimize the condition number $\kappa(V)$ of $V$, which is defined as

$$
\kappa(V)=\sqrt{\frac{\sigma_{\max }}{\sigma_{\min }}}
$$

265

where $\sigma_{\max }$ and $\sigma_{\min }$ are the largest and smallest eigenvalues of $V^{*} V$, respectively. To this aim we need to introduce the wrap-around distance on the unit interval, that is

$$
d_{w}(f, g)=\inf _{q \in \mathbb{Z}}|f-g+q| .
$$

If we define the minimal separation between the $f_{n}$ as

$$
\Delta=\min _{n, n^{\prime}=0, \cdots, N-1, n \neq n^{\prime}} d_{w}\left(f_{n}, f_{n^{\prime}}\right),
$$

then we know from [18] (see the appendix A) that for $M>1 / \Delta+1$ the condition number $\kappa(V)$ admits the upper bound

$$
\kappa(V) \leq \sqrt{\frac{M+1 / \Delta-1}{M-1 / \Delta-1}} .
$$

We observe that such upper bound is a decreasing function of $M$ and of $\Delta$. In particular, $\kappa(V)$ tends to the optimal value 1 when $M$ tends to $+\infty$. It is then tempting to choose $M$ and $\Delta$ large. Let us see how to maximize $\Delta$. 
The following lemma specifies the minimal separation between the $f_{n}$ in the case there exist at least two propagating guided modes, that is $k>\pi / d$, or equivalently, $\lambda<2 d$.

Lemma 2 For $\delta \leq \lambda$, we have

$$
\Delta=\left(1-\sqrt{1-\frac{\lambda^{2}}{4 d^{2}}}\right) \frac{\delta}{\lambda}
$$

Proof : From the expression of the $f_{n}$ given by $(18)$, we clearly have

$$
\min _{n, n^{\prime}=0, \cdots, N-1, n \neq n^{\prime}} d_{w}\left(f_{n}, f_{n^{\prime}}\right)=\min \left(d_{w}\left(f_{0}, f_{1}\right), d_{w}\left(f_{N-1}, f_{0}\right)\right),
$$

and

$$
\begin{gathered}
d_{w}\left(f_{0}, f_{1}\right)=\left(1-\sqrt{1-\frac{\lambda^{2}}{4 d^{2}}}\right) \frac{\delta}{\lambda} \\
d_{w}\left(f_{N-1}, f_{0}\right)=1-\left(1-\sqrt{1-(N-1)^{2} \frac{\lambda^{2}}{4 d^{2}}}\right) \frac{\delta}{\lambda},
\end{gathered}
$$

with $N=[2 d / \lambda]$ and [.] means the floor function. We observe that $d_{w}\left(f_{0}, f_{1}\right)$ is an increasing function of $\delta \in[0, \lambda]$ while $d_{w}\left(f_{N-1}, f_{0}\right)$ is a decreasing function of $\delta$. We obtain the claimed result if for $\delta=\lambda$, then $d_{w}\left(f_{0}, f_{1}\right) \leq d_{w}\left(f_{N-1}, f_{0}\right)$. This amounts to prove that

$$
\sqrt{1-\frac{\lambda^{2}}{4 d^{2}}}+\sqrt{1-\left(\left[\frac{2 d}{\lambda}\right]-1\right)^{2} \frac{\lambda^{2}}{4 d^{2}}} \geq 1
$$

Denoting $x=\lambda / 2 d \in[0,1]$, we have

$$
\sqrt{1-x^{2}}+\sqrt{1-\left(\left[\frac{1}{x}\right]-1\right)^{2} x^{2}} \geq 1-x^{2}+1-\left(\left[\frac{1}{x}\right]-1\right)^{2} x^{2} .
$$

By using the fact that

$$
0 \leq\left[\frac{1}{x}\right]-1 \leq \frac{1}{x}-1
$$

we obtain that

$$
\sqrt{1-x^{2}}+\sqrt{1-\left(\left[\frac{1}{x}\right]-1\right)^{2} x^{2}} \geq 1+2 x-2 x^{2} \geq 1+2 x-2 x=1,
$$

which completes the proof.

From the above lemma we conclude that the value of $\delta$ which maximizes the minimal separation between the $f_{n}$ is $\delta=\lambda$, and the minimal separation (21) becomes in this case

$$
\Delta=1-\sqrt{1-\frac{\lambda^{2}}{4 d^{2}}}
$$


In conclusion we must use a large number of sources and receivers separated by a distance which coincides with the wavelength. To illustrate the above analysis we have plotted in the figure 3 the log of the condition number $\kappa(V)$ as a function of $\delta / \lambda$ for $N=8$ and for increasing values of $M$, that is $M=N, 2 N, 4 N, 8 N$. The peaks on figure 3 for $\delta>\lambda$ reveal the cases where $V^{*} V$ is not invertible, which confirms that the choice $\delta / \lambda=1$ is relevant. The figure 3 also shows that the conditioning of $V$ is improving for increasing $M$. In the figure 4 we compare the $\log$ of $\kappa(V)$ and the log of the upper bound given by (22) for all values of $M$, when $N=8$ and $\delta=\lambda$. This picture confirms that $\kappa(V) \rightarrow 1$ when $M \rightarrow+\infty$.

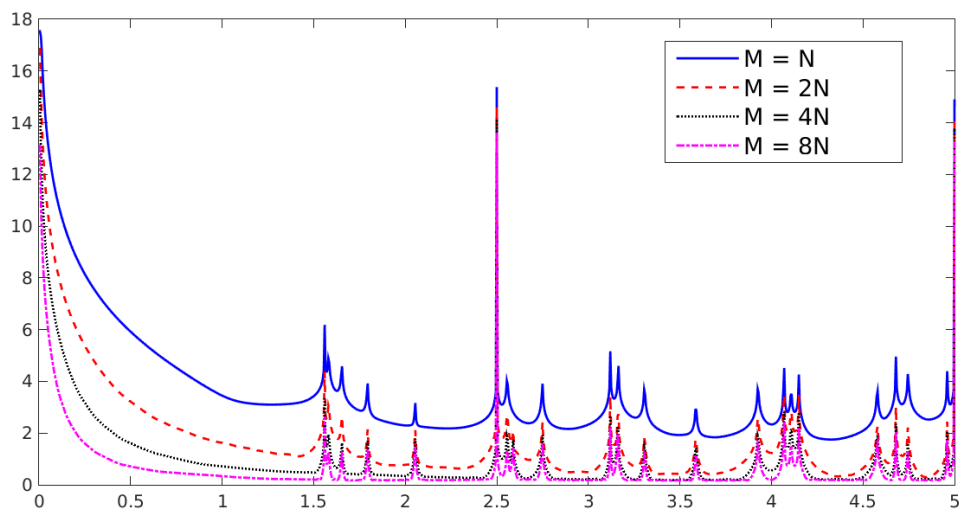

Figure 3: The log of the condition number $\kappa(V)$ as a function of $\delta / \lambda$

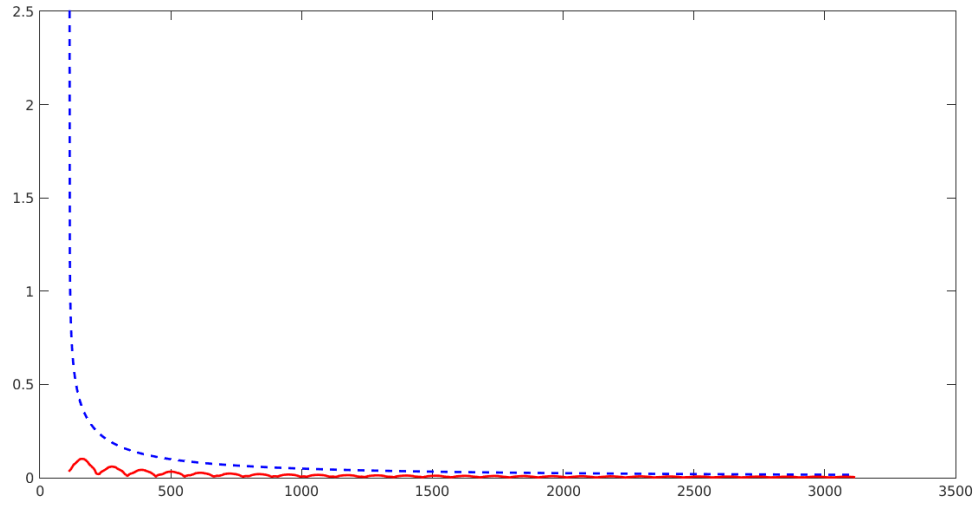

Figure 4: Comparison between the log of the condition number $\kappa(V)$ (solid line) and of the upper bound given by 22 (dashed line) for all $M$ 


\section{Surface data in the time domain}

We now come back to the initial problem (1). By defining the Fourier trans-

305 form of $v(x, t)$ with respect to time $t$ by

$$
\widehat{v}(x, \omega)=\int_{\mathbb{R}} v(x, t) e^{+i \omega t} d t
$$

where $v$ is the solution to problem (1), it can be shown that for any fixed $\omega>0$ the solution $u=\widehat{v}(\cdot, \omega)$ satisfies the problem (11) with

$$
k=\omega / c, \quad \phi=\widehat{\chi}(\omega) f .
$$

In particular, it is fundamental to consider the exponential $e^{+i \omega t}$ and not $e^{-i \omega t}$ in the definition (23) of the Fourier transform in order that $u$ satisfies the radiation condition $(R C)$ in problem (11) (see for example [19]). For some space function $g\left(x_{1}\right)$ and some time function $\chi(t)$, we obtain the measurement matrix $\mathcal{M}(\omega)$ at frequency $\omega$ as follows: we compute the Fourier transform at such frequency of the solution $v$ to problem (1) which corresponds to Neumann data

$$
f\left(x_{1}\right) \chi(t)=g\left(x_{1}-x_{1}^{s \pm}\right) \chi(t) / \widehat{\chi}(\omega)
$$

at all receivers $x_{1}^{r \pm}$ by using 23 , for all sources $x_{1}^{s \pm}$. We then subtract to the 315

of the Fourier transform of all sources $x_{1}^{s \pm}$ to the same problem as (1) where no boundary condition on $\partial D$ is prescribed. We hence obtain the scattered fields in the frequency domain and are in a position to use the method described in the previous section. In conclusion, for each $\omega$ we obtain the LSM matrix $\mathcal{U}(\omega)$ and then an indicator function $\psi(\cdot, \omega)$ of the defect.

An important question is now: how can we exploit the indicator functions $\psi(\cdot, \omega)$ at different frequencies $\omega \in\left(\omega_{-}, \omega_{+}\right)$in order to obtain the best possible global indicator function $\Psi$ ? In 20 , the authors propose two different strategies in the case of free space: they compute either a "serial" or "parallel" indicator 325 function, respectively defined in the sampling grid $G$ by

$$
\Psi^{s}=\left(\int_{\omega_{-}}^{\omega_{+}}|\psi(\cdot, \omega)|^{-2} d \omega\right)^{-1 / 2}, \quad \Psi^{p}=\left(\int_{\omega_{-}}^{\omega_{+}}|\psi(\cdot, \omega)|^{2} d \omega\right)^{1 / 2} .
$$

The authors also establish a relationship between the first one and the Linear Sampling Method in the time domain. In our numerical experiments we will use the first one since it gives slightly better results on the whole. In addition, in order to take into account a scaling factor between the different indicator 330 functions $\psi(\cdot, \omega)$ for a large spectrum of frequencies, instead of the previous function $\Psi^{s}$ we consider

$$
\Psi^{s}=\left(\int_{\omega_{-}}^{\omega_{+}} \frac{\max _{z \in G}|\psi(z, \omega)|^{2}}{|\psi(\cdot, \omega)|^{2}} d \omega\right)^{-1 / 2}
$$


Let us now specify the functions $g$ and $\chi$ that we will use in the numerical experiments. The function $g$ is chosen as a triangular function of integral 1 given by

$$
g\left(x_{1}\right)=\frac{10}{d} \max \left(1-\frac{10\left|x_{1}\right|}{d}, 0\right),
$$

while the function $\chi(t)$ is chosen as a sum of functions

$$
\chi_{n}(t)=\frac{d \cdot}{d t}\left[\sin \left(A_{n} t\right) e^{-B_{n}\left(t-C_{n}\right)^{2}}\right],
$$

where the real numbers $A_{n}, B_{n}, C_{n}$ can be fixed in order to select the mean frequency and the support of the signal in the frequency domain. Considering the sequence of frequencies $\omega_{n}=\pi c n / d$ for $n \in \mathbb{N}$, we set $A_{n}, B_{n}, C_{n}$ such that the mean frequency of $\widehat{\chi_{n}}(\omega)$, denoted by $\omega_{\text {mean }, n}$, coincides with the mean value of two successive frequencies $\omega_{n-1}$ and $\omega_{n}$, that is $\omega_{\text {mean }, n}=\frac{\pi c(n-0.5)}{d}$, and such that the support of $\widehat{\chi_{n}}(\omega)$ is mainly contained in $\left[\omega_{n-1}, \omega_{n}\right]$. This is obtained by choosing

$$
B_{n}=\frac{\pi^{2}}{200 d^{2}}, \quad C_{n}=\frac{5}{\sqrt{2 B_{n}}}, \quad A_{n}=\omega_{\text {mean }, n}-\frac{4 B_{n}}{\omega_{\text {mean }, n}} .
$$

Throughout the paper we set $c=1$ and $d=1$. For a given $N$, the corresponding function $\chi_{N}$ is such that the support of $\widehat{\chi_{N}}$ doesn't contain any of the cut345 off frequencies $\omega_{n}, n \in \mathbb{N}$, which correspond to vanishing group velocities $\beta_{n}$ from (7). Since these cut-off frequencies are avoided, this enables us to obtain some scattered fields in the time domain that decrease quite rapidly to 0 when $t \rightarrow+\infty$ and then to bound by a reasonable time the total duration of the forward computation that provides the synthetic data. In the picture 5 we have plotted the function $\chi_{12}(t)$ as well as its Fourier transform $\widehat{\chi_{12}}(\omega)$. In the following numerical experiments we will use a combination of several $\chi_{n}$ functions, namely

$$
\chi(t)=\sum_{n=1}^{12} \chi_{n}(t) .
$$

In order to illustrate the need for the support of $\widehat{\chi}$ to exclude the cut-off frequencies $\omega_{n}$, the obstacle $D$ being the square represented on figure 7, we consider the solution to problem (1) obtained with the time domain code described in section 5.1 for the space function $f=g\left(\cdot-x_{1}\right)$ located on $\Gamma_{d}$ at $x_{1}=-R$ and two kinds of time functions $\chi$. The first one is the function $\chi$ given by $(28)$ while the second one is given by $\rho: t \mapsto d^{2}\left(e^{-(t-D)^{2} / E}\right) / d t^{2}$ for $D=5 / 4 \sqrt{2}$ and $E=5 / 16$. The support of $\hat{\rho}$ contains several cut-off frequencies. On the figure 6 we have represented the solution with respect to time at two receivers on $\Gamma_{d}$ : the first one is located at $x_{1}=-R$ while the second one is located at $x_{1}=R+(M-1) \delta$. We can observe that with the time function $\chi$ the scattered signal vanishes much more rapidly than with $\rho$. 

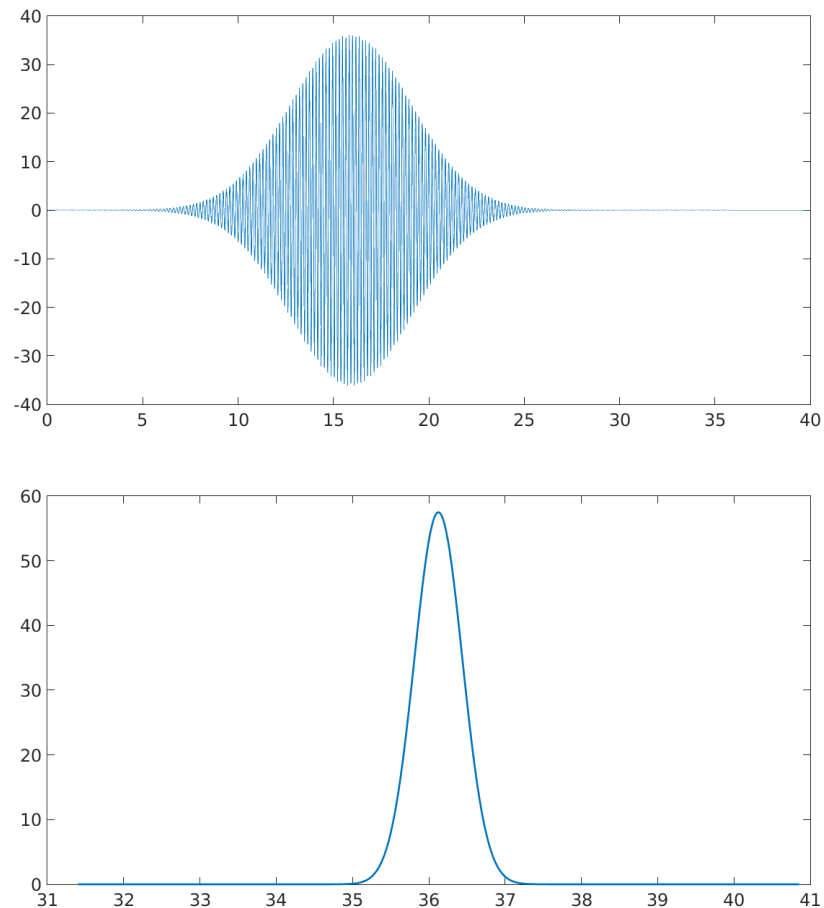

Figure 5: Top: the localized function $\chi_{12}$ with respect to time $t$. Bottom: its Fourier transform $\widehat{\chi 12}$ with respect to frequency $\omega$.

\section{Numerical experiments}

365

\subsection{Forward implementation}

In what follows we will compute some synthetic data by solving either the forward problem in the frequency domain (11) or the forward problem in the time domain (1). The problem (11) is solved by using a frequency domain code. It is based on a classical Lagrange Finite Element Method in a domain which is bounded by two artificial transverse sections and by using a DirichletTo-Neumann operator on each of them (see [1). The problem (1) is solved by using a time domain code. It is based on a Finite Element Method for the space variable and a Finite Difference Scheme for the time variable. More precisely, while a classical leap-frog scheme is used for the time variable, a 375 spectral finite element method is used for the space variable in order to benefit from two advantages: low dispersion and mass lumping (see for example 21] for a precise analysis of such a method). The domain is bounded by two Perfectly Matched Layers (see for example [22]), the second-order equation is split into two first-order equations in the PMLs while some Mortar elements are used at 380 the interface between the reference medium and the PMLs. The scattered fields in the time domain that result from the time domain code are transformed into 

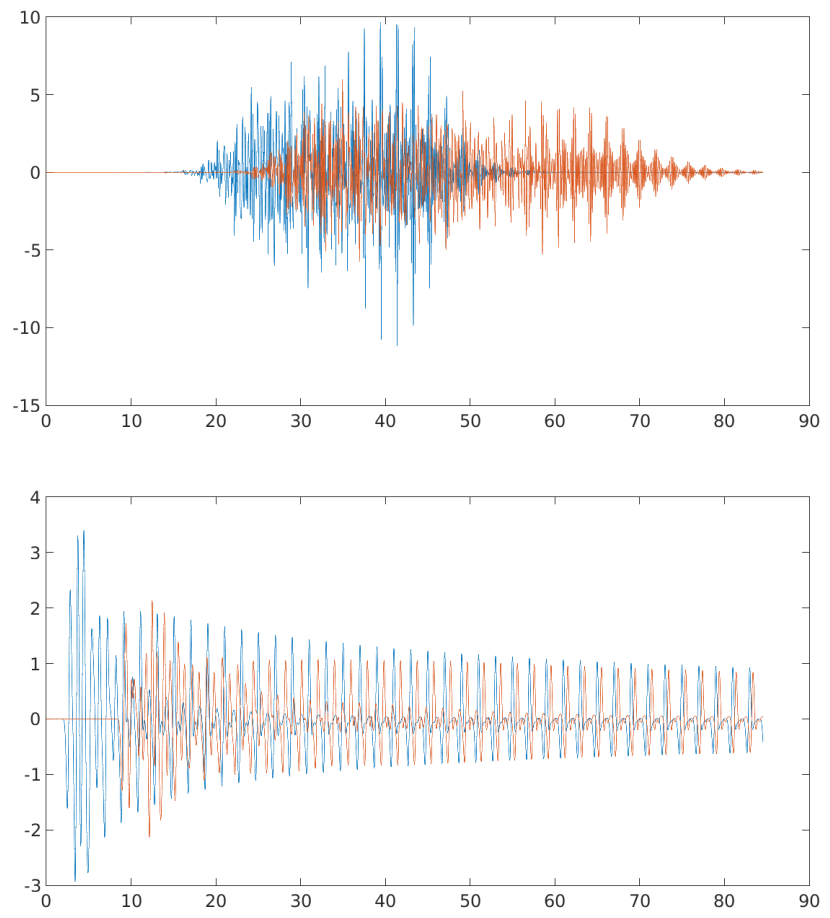

Figure 6: Top: scattered field for the localized function $\chi$ with respect to time $t$. Bottom: scattered field for the non localized function $\rho$ with respect to time $t$.

scattered fields in the frequency domain with the help of a classical Fast Fourier Transform computation.

\subsection{Numerical results}

We now present some numerical experiments for two different obstacles $D$ : a square and the union of two rectangles (see figures 7 and 8 , respectively). The constant $R$ involved in the Linear Sampling Method (9) is set to $R=1$, the sampling grid is also limited by the transverse sections $\Sigma_{-R}$ and $\Sigma_{R}$. In all the following numerical experiments, the number of sources and receivers $2 M$ and the distance $\delta$ between two sources/receivers are calibrated on the highest frequency that we consider for each inverse problem. More precisely, if $N$ and $\lambda$ denote the number of propagating modes and the wavelength corresponding to such frequency, the number of sources and receivers is such that $M=3 N$ and the distance between two sources/receivers is $\delta=\lambda$, as recommended in section

395 3.3. In what follows we compare four kinds of computations.

1. The artificial data are obtained by solving problem (11) with the help of the frequency domain code with $\phi\left(x_{1}\right)=g\left(x_{1}-x_{1}^{s \pm}\right)$ (in view of 24) and (25)) for the space function $g$ given by (27) at a fixed frequency 
$\omega$ corresponding to $N=4, N=8$ and $N=12$ propagating modes, respectively, and the inverse problem is solved at such a fixed frequency. The results are given on figure 7 for the square and on figure 8 for the union of two rectangles.

2. The artificial data are obtained by solving problem (1) with the help of the time domain code for $f \chi$ given by 25 for the space function $g$ given by (27) and the time function given by (28) (see section 4).

Three cases are analyzed.

(a) The inverse problem is solved at a single frequency given by the mean frequency of the function $\chi_{N}$, which corresponds to $N$ propagating modes, for $N=4, N=8$ and $N=12$. The results are given on figure 9 for the square and on figure 10 for the union of two rectangles.

(b) The inverse problem is solved for the set of frequencies $\omega$ that support the function $\chi_{N}$ by using the indicator function 26 for $\omega_{-}=\omega_{N-1}$ and $\omega_{+}=\omega_{N}$, all of these frequencies corresponding to the same number $N$ of propagating modes, for $N=4, N=8$ and $N=12$. The results are given on figure 11 only for the union of two rectangles.

(c) The inverse problem is solved for the set of all frequencies $\omega$ that support one of the functions $\chi_{N}, N=8, \cdots, 12$, by using the sum of the indicator functions 26 in each of the intervals $\left[\omega_{N-1}, \omega_{N}\right]$ for $N=8, \cdots, 12$, the frequencies in $\left[\omega_{N-1}, \omega_{N}\right]$ corresponding to $N$ propagating modes. The results are given on figure 12 for the square and for the union of two rectangles.

Case 1. serves as a reference successful case. A comparison between the numerical experiments of 1 . and 2(a). shows a slight degradation in the second case. Essentially, this is due to the fact that the artificial data in the time domain are less accurate than those obtained in the frequency domain and to the bad conditioning of the Vandermonde matrix $V$ we have to invert. A comparison between the numerical experiments of 2(a). and 2(b). shows an improvement due to the use of several frequencies instead of only one, even if these frequencies correspond to the same number of propagating modes. Lastly, a comparison between the numerical experiments of 2(b). and 2(c). shows that if we use several frequencies corresponding to several numbers of propagating modes $N$ (provided $N$ is not too small), then we go on improving the quality of the identification.

We complete this numerical section with a brief sensitivity analysis of two parameters: the amount of sources/receivers and the amplitude of the noise 435 corrupting the time domain data. In order to illustrate the importance of using a sufficiently large amount of sources and receivers, we compare on figure 13 the results of figure 9 for $N=12$ when $M=N$ and $M=2 N$ instead of $M=3 N$. In order to illustrate the impact of noise, we perturb the time signal at each point $x_{1}^{m \pm}, m=0, \cdots, M-1$ by a pointwise gaussian noise so that 440 the corresponding relative $L^{2}$ norm of the noise function be $\sigma$. The results of figure 12 correspond to the time domain data which directly comes out from the time domain code and thus to noise free data. We compare on figure 14 these results for the union of two rectangles in the $2(\mathrm{c})$. case with the results 
obtained for noisy data of amplitude $\sigma=0.01, \sigma=0.1$ and $\sigma=0.5$. Increasing the amplitude of noise slightly decreases the quality of the identification in the sense that the ratio between the highest and the lowest values of the function $\Psi^{s}$ given by 26 decreases. Visually, the identification remains satisfactory.
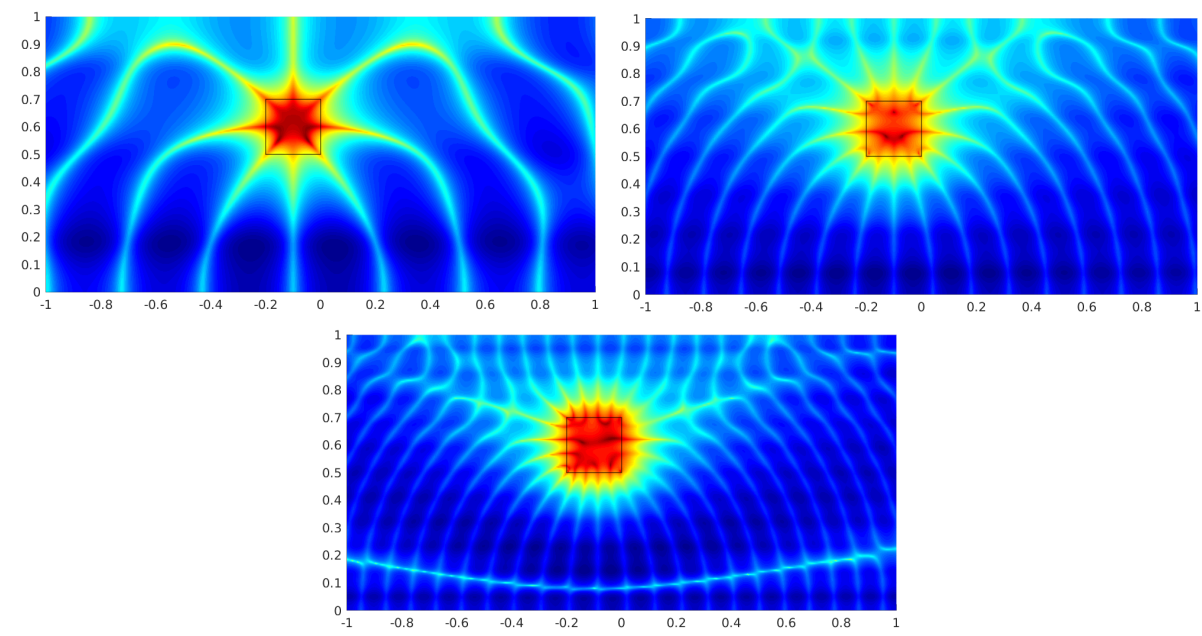

Figure 7: Frequency domain data. Top left: $N=4$. Top right: $N=8$. Bottom: $N=12$.
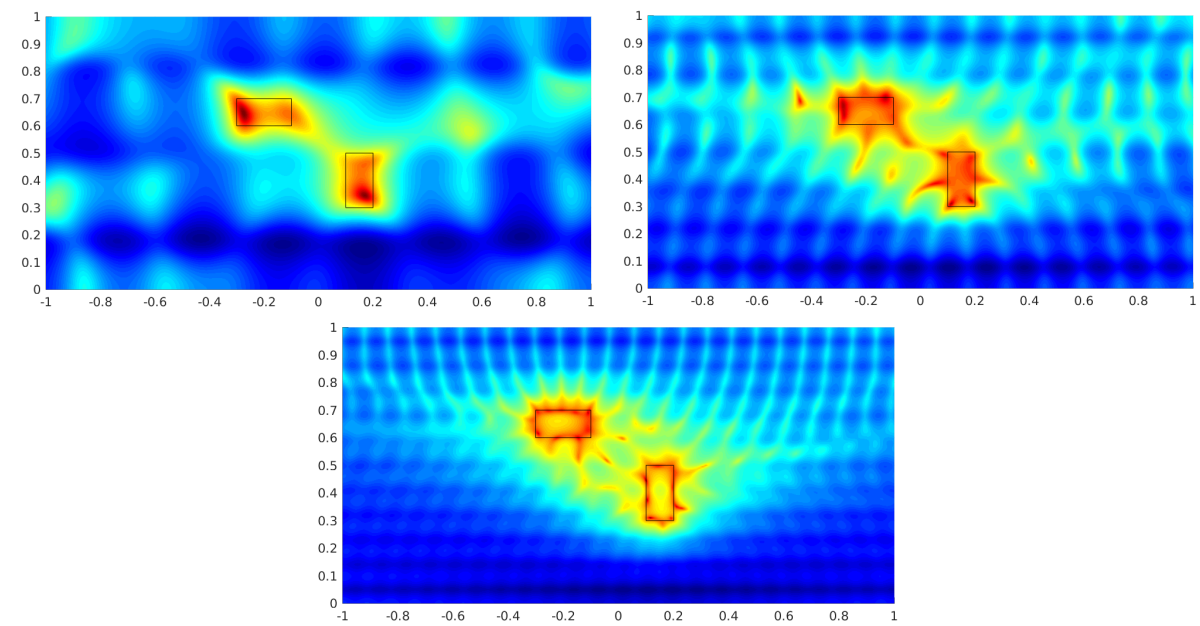

Figure 8: Frequency domain data. Top left: $N=4$. Top right: $N=8$. Bottom: $N=12$. 

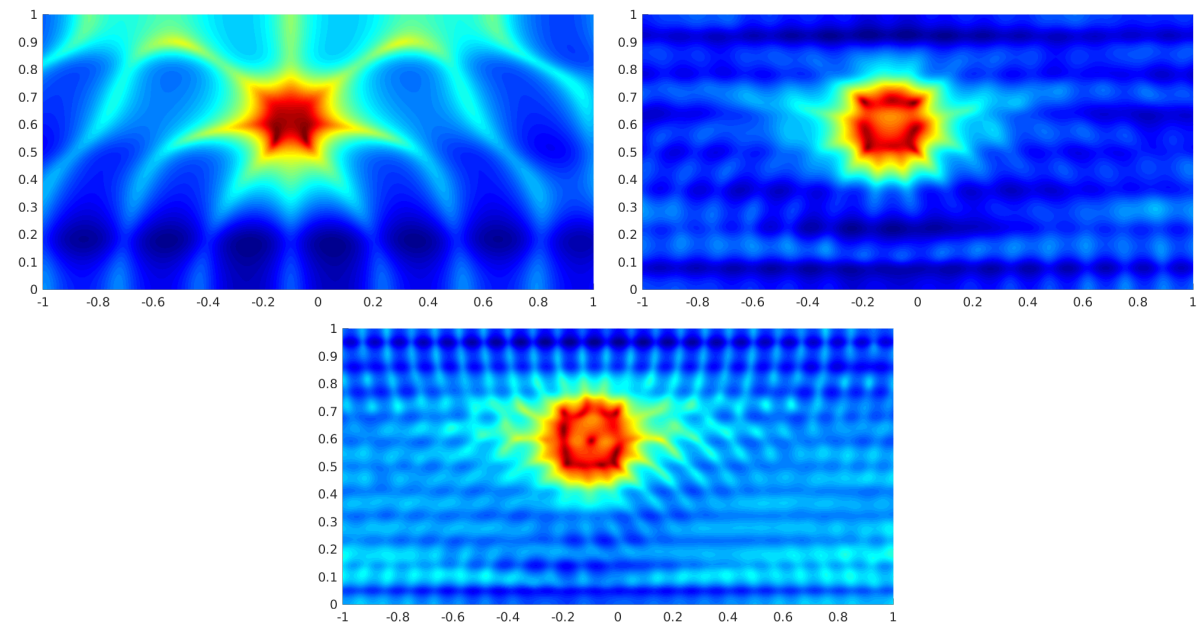

Figure 9: Time domain data with $\chi=\sum_{n=1}^{12} \chi_{n}$ and single frequency corresponding to $N$ propagating modes. Top left: $\mathrm{N}=4$. Top right: $\mathrm{N}=8$. Bottom: $\mathrm{N}=12$.
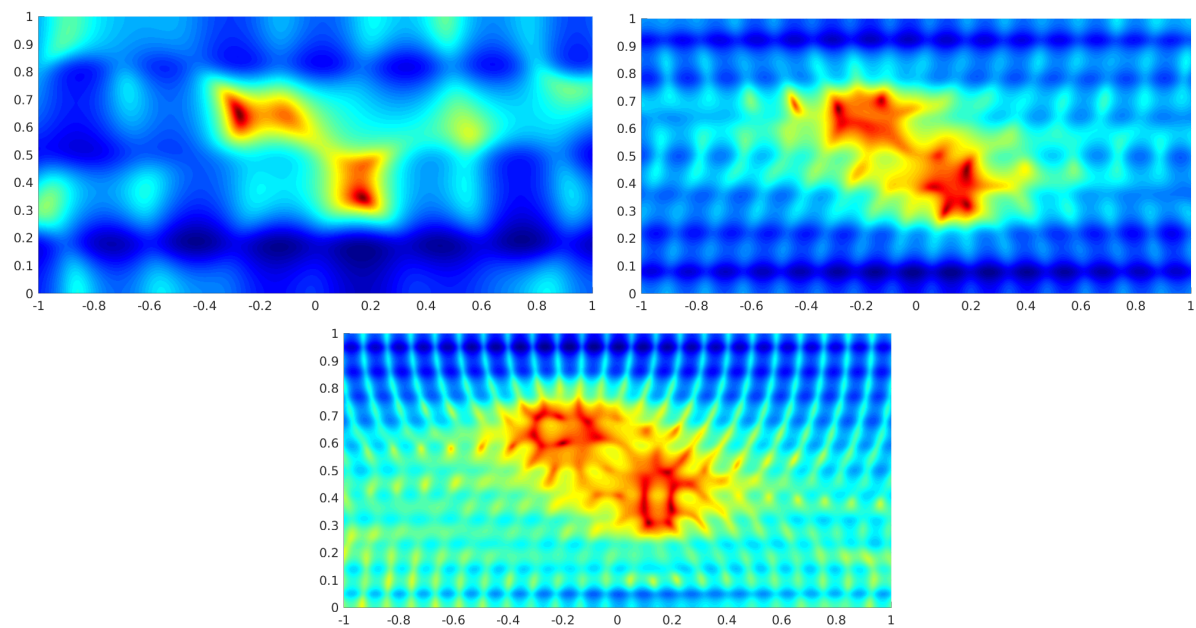

Figure 10: Time domain data with $\chi=\sum_{n=1}^{12} \chi_{n}$ and single frequency corresponding to $N$ propagating modes. Top left: $\mathrm{N}=4$. Top right: $\mathrm{N}=8$. Bottom: $\mathrm{N}=12$.

\section{Conclusions and perspectives}

We have shown in this paper that imaging defects in an acoustic waveguide from surface data in the time domain is feasible with the help of the Linear Sampling Method in the frequency domain. This requires to use a sufficiently large number of sources/receivers and an appropriate distance between them. We also have to calibrate the time shape of the incident wave in order to bound 455 the time support of the scattered field as much as possible. Lastly, increasing 

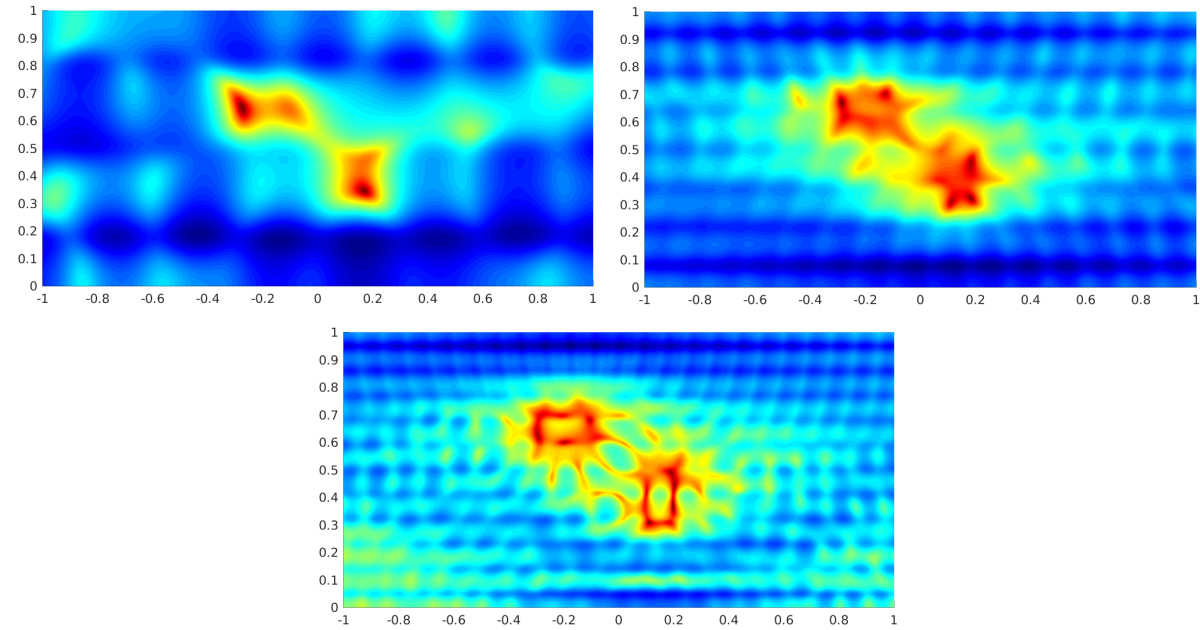

Figure 11: Time domain data with $\chi=\sum_{n=1}^{12} \chi_{n}$ and multiple frequencies in the support of $\chi_{N}$. Top left: $\mathrm{N}=4$. Top right: $\mathrm{N}=8$. Bottom: $\mathrm{N}=12$.
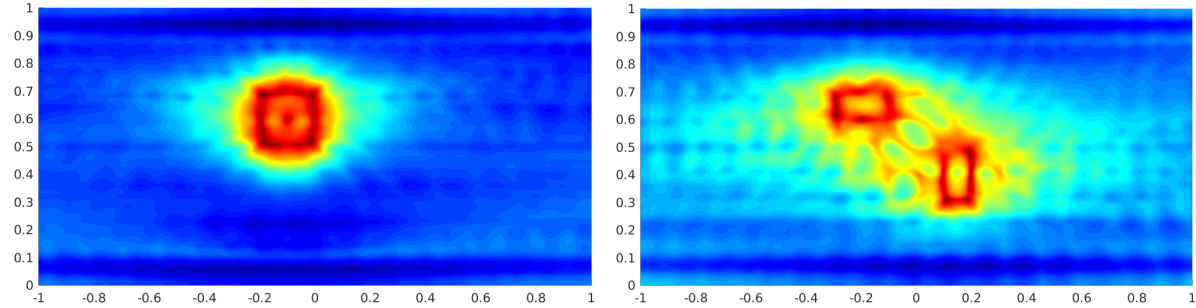

Figure 12: Time domain data with $\chi=\sum_{n=1}^{12} \chi_{n}$ and multiple frequencies in the union of the supports of $\chi_{n}, n=8, \cdots, 12$.

the number of frequencies used in the inversion scheme, in particular the largest ones, enables us to improve the quality of the identification.

It is natural to compare our approach with the one proposed in [15. While in 15] the time domain data are treated as such, in our paper they are transformed to the frequency domain in order to make use of a modal description of the waveguide. The main difference concerns regularization. In [15, inverting the space/time operator at each sampling point leads to a large ill-posed system which is solved with Tikhonov regularization. In contrast, for all frequencies we invert a space operator at each sampling point and our "physical" regularization of the corresponding system consists in limiting the data to the contribution of propagating modes, the number of which increases with respect to the frequency. This leads to very small systems to invert. From the numerical point of view it is hard to compare the efficiency of our two approaches since our data are not the same, in particular the mean frequencies of our signals are higher than those used in [15]. But a systematic comparison with the same data would be 

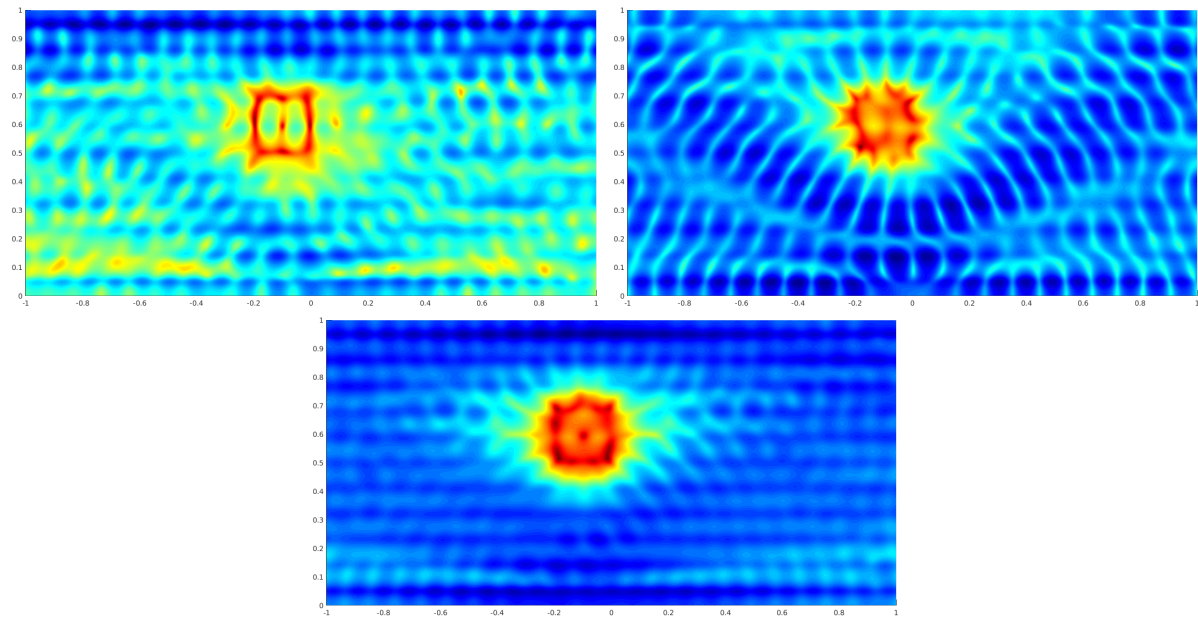

Figure 13: Time domain data with $\chi=\sum_{n=1}^{12} \chi_{n}$ and single frequency corresponding to $N=12$ propagating modes. Top left: $M=N$. Top right: $M=2 N$. Bottom: $M=3 N$.
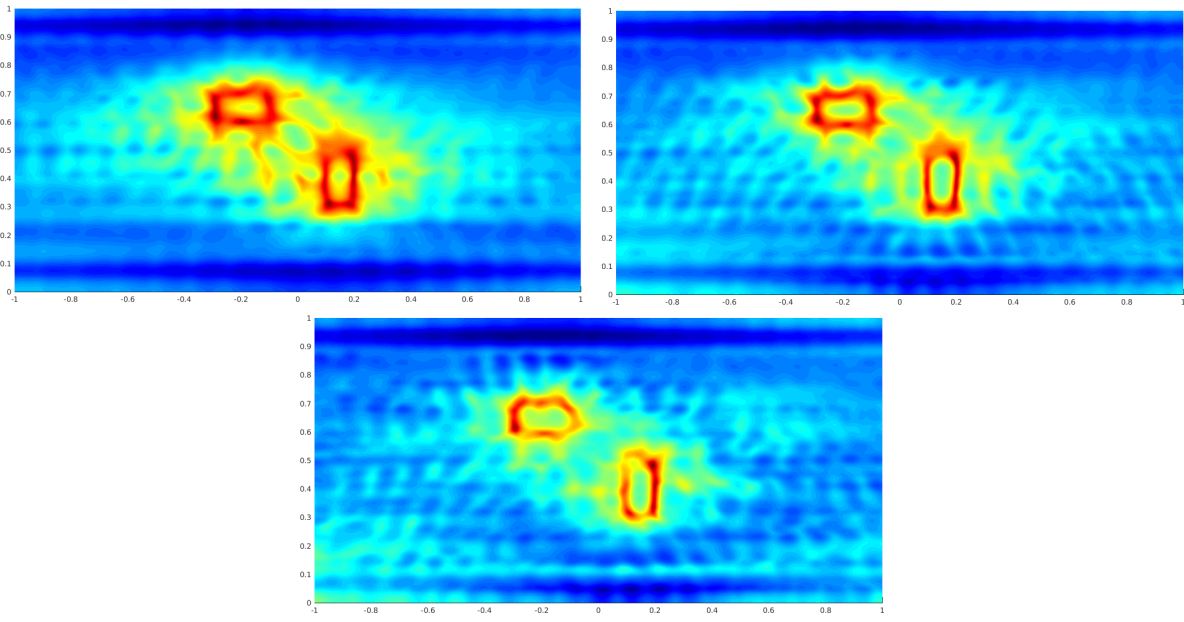

Figure 14: Time domain data with $\chi=\sum_{n=1}^{12} \chi_{n}$ and multiple frequencies in the union of the supports of $\chi_{n}, n=8, \cdots, 12$. Top left: $\sigma=0.01$. Top right: $\sigma=0.1$. Bottom: $\sigma=0.5$.

\section{interesting.}

Our aim is now to extend our method to the case of elasticity, since many ultrasonic Non Destructive Testing applications concern elastic waveguides. In particular, we intend to try our method from experimental data instead of synthetic data, which is quite challenging. 


\section{Appendix A: the conditioning of the Vandermonde matrices with unit complex entries}

The objective of this appendix is to recall some more or less recent results concerning the condition number of the Vandermonde matrices with entries on the unit circle. This subject is of importance in the field of signal processing and a number of contributions are dedicated to it. We are interested in the rectangular Vandermonde matrices of type

$$
V=\left[\begin{array}{cccc}
1 & 1 & \cdots & 1 \\
e^{2 \pi i f_{0}} & e^{2 \pi i f_{1}} & \cdots & e^{2 \pi i f_{N-1}} \\
\cdots & \cdots & \cdots & \cdots \\
e^{2 \pi i(M-1) f_{0}} & e^{2 \pi i(M-1) f_{1}} & \cdots & e^{2 \pi i(M-1) f_{N-1}}
\end{array}\right],
$$

where the $f_{n}, n=0, \cdots, N-1$ are real numbers and $M \geq N$. It is well known that for $M=N$, the determinant of the square matrix $V$ is given by

$$
\operatorname{det}(V)=\prod_{n \neq n^{\prime}}\left(e^{2 \pi i f_{n}}-e^{2 \pi i f_{n^{\prime}}}\right),
$$

so that $V$ is invertible if and only if for all $n^{\prime} \neq n, f_{n^{\prime}}-f_{n}$ is not an integer. We deduce from the case when $M=N$ that in the more general case when $M \geq N$, if for all $n^{\prime} \neq n, f_{n^{\prime}}-f_{n}$ is not an integer, the matrix $V$ is injective, so that $V^{*} V$ is invertible.

Now let us consider the conditioning of the matrix $V$. Let us denote $\kappa(V)$ its condition number. For $n, n^{\prime}=0, \cdots, N-1$, we have

$$
\begin{gathered}
\left(V^{*} V\right)_{n n^{\prime}}=\sum_{m=0}^{M-1} e^{-2 \pi i\left(f_{n}-f_{n^{\prime}}\right) m} \\
=\left\{\begin{array}{ccc}
M & \text { if } & f_{n}-f_{n^{\prime}} \in \mathbb{Z} \\
\frac{1-e^{-2 \pi i\left(f_{n}-f_{n^{\prime}}\right) M}}{1-e^{-2 \pi i\left(f_{n}-f_{n^{\prime}}\right)}} & \text { if } & f_{n}-f_{n^{\prime}} \notin \mathbb{Z} .
\end{array}\right.
\end{gathered}
$$

Hence we observe that if $M / N$ is a positive integer and the $f_{n}$ are uniformly spread on the unit segment $[0,1]$, namely $f_{n}=n / N, n=0, \cdots, N-1$, then

$$
V^{*} V=M I_{N},
$$

where $I_{N}$ is the $N \times N$ identity matrix, which means that $V$ has the best possible condition number, that is $\kappa(V)=1$. In the general case, to our best knowledge a simple expression of $\kappa(V)$ in terms of $M$ and the $f_{n}, n=0, \cdots, N-1$ is unknown. However, an interesting upper bound for $\kappa(V)$ was established very recently in 18 . We give the proof for the sake of completeness and to clarify some details that are not given in [18.

Theorem If the minimal separation $\Delta$ defined by (21) satisfies $M>1 / \Delta+1$, then

$$
\kappa(V) \leq \sqrt{\frac{M+1 / \Delta-1}{M-1 / \Delta-1}} .
$$


Proof: We introduce the Beurling function

$$
B(t)=\left(\frac{\sin \pi t}{\pi}\right)^{2}\left(\sum_{j=0}^{+\infty} \frac{1}{(t-j)^{2}}-\sum_{j=-\infty}^{-1} \frac{1}{(t-j)^{2}}+\frac{2}{t}\right),
$$

which is such that the function $(B-\operatorname{sgn})$ is integrable and satisfies

$$
B(t)-\operatorname{sgn}(t) \geq 0, \forall t \in \mathbb{R}, \quad \int_{-\infty}^{+\infty} B(t)-\operatorname{sgn}(t) d t=1 .
$$

505

We also introduce, for an interval $[a, b]$ and $\delta>0$, the Selberg majorant and minorant

$S_{+}(t)=\frac{1}{2} B(\delta(t-a))+\frac{1}{2} B(\delta(b-t)), \quad S_{-}(t)=-\frac{1}{2} B(\delta(a-t))-\frac{1}{2} B(\delta(t-b))$.

These two functions $S_{ \pm}$are integrable and satisfy

$$
S_{-}(t) \leq \chi_{[a, b]}(t) \leq S_{+}(t), \forall t \in \mathbb{R}, \quad \int_{-\infty}^{+\infty} S_{ \pm}(t) d t=b-a \pm \frac{1}{\delta},
$$

where $\chi_{[a, b]}$ is the indicator function of set $[a, b]$. Moreover, the Fourier transforms of $B$ and $S_{ \pm}$satisfy $\widehat{B}(x)=0$ for $|x| \geq 1$ and $\widehat{S}_{ \pm}(x)=0$ for $|x| \geq \delta$, 510 which in particular implies that $B$ and $S_{ \pm}$are infinitely smooth. Here the Fourier transform is defined by

$$
\widehat{f}(x)=\int_{\mathbb{R}} f(t) e^{-2 \pi i x t} d t .
$$

The above properties of the functions $B$ and $S_{ \pm}$are well-known (see for example [23]). We will need the additional following estimate: there exists $C>0$ (depending on $a, b$ and on $\delta$ ) such that

$$
\left|S_{ \pm}(t)\right| \leq \frac{C}{1+t^{2}}, \quad \forall t \in \mathbb{R} .
$$

515 We first prove that there exists some $c>0$ such that

$$
0 \leq B(t)-\operatorname{sgn}(t) \leq \frac{c}{1+t^{2}}, \quad \forall t \in \mathbb{R} .
$$

From the expression of $B$ and the classical identity

$$
\sum_{j=-\infty}^{+\infty} \frac{1}{(t-j)^{2}}=\left(\frac{\pi}{\sin \pi t}\right)^{2}
$$

we obtain the expression

$$
B(t)=1+2\left(\frac{\sin \pi t}{\pi}\right)^{2}\left(\frac{1}{t}-\sum_{j=1}^{+\infty} \frac{1}{(t+j)^{2}}\right),
$$


which we need for $t>0$. It happens that

$$
\frac{1}{t+1}=\int_{1}^{+\infty} \frac{1}{(t+u)^{2}} d u \leq \sum_{j=1}^{+\infty} \frac{1}{(t+j)^{2}} \leq \int_{0}^{+\infty} \frac{1}{(t+u)^{2}} d u=\frac{1}{t},
$$

which implies (34) for $t>0$. For $t<0$ we use the expression

$$
B(t)=-1+2\left(\frac{\sin \pi t}{\pi}\right)^{2}\left(\frac{1}{t}+\sum_{j=0}^{+\infty} \frac{1}{(t-j)^{2}}\right) .
$$

$$
-\frac{1}{t}=\int_{0}^{+\infty} \frac{1}{(t-u)^{2}} d u \leq \sum_{j=0}^{+\infty} \frac{1}{(t-j)^{2}} \leq \frac{1}{t^{2}}+\int_{0}^{+\infty} \frac{1}{(t-u)^{2}} d u=\frac{1}{t^{2}}-\frac{1}{t}
$$

which implies (34) for $t<0$. To prove 33 , we remark that

$$
\begin{gathered}
S_{+}(t)=\frac{1}{2}(B(\delta(t-a))-\operatorname{sgn}(\delta(t-a)))+\frac{1}{2}(B(\delta(b-t))-\operatorname{sgn}(\delta(b-t))) \\
+\frac{1}{2}(\operatorname{sgn}(\delta(t-a))+\operatorname{sgn}(\delta(b-t))) .
\end{gathered}
$$

We then apply (34) to the first two functions while the third one vanishes outside $[a, b]$. The proof is the same for $S_{-}$.

Let us choose $a=0, b=M-1$ and $\delta=\Delta$ in the definition of $S_{ \pm}$. Then we consider the function $v(x)=\sum_{n=0}^{N-1} u_{n} e^{-2 \pi i f_{n} x}$ for any $x \in \mathbb{R}$ and any vector $U=\left(u_{0}, u_{1}, \cdots, u_{N-1}\right) \in \mathbb{C}^{N}$. On the one hand we have

$$
\sum_{m=0}^{M-1}|v(m)|^{2}=\sum_{n=0}^{N-1} \sum_{n^{\prime}=0}^{N-1} \sum_{m=0}^{M-1} u_{n} \bar{u}_{n^{\prime}} e^{-2 \pi i\left(f_{n}-f_{n^{\prime}}\right) m},
$$

which by using $(30)$ implies

$$
\sum_{m=0}^{M-1}|v(m)|^{2}=\sum_{n=0}^{N-1} \sum_{n^{\prime}=0}^{N-1}\left(V^{*} V\right)_{n n^{\prime}} u_{n} \bar{u}_{n^{\prime}}=\|V U\|^{2} .
$$

On the other hand we have

$$
\sum_{m=0}^{M-1}|v(m)|^{2}=\left\langle\chi_{[0, M-1]} D,|v|^{2}\right\rangle
$$

530 where $D$ is the Dirac comb $D=\sum_{l \in \mathbb{Z}} \delta_{l}$ and the brackets mean the duality pairing between the compactly supported distribution $\chi_{[0, M-1]} D$ and the $C^{\infty}$ function $\psi: x \mapsto|v(x)|^{2}$. We note that the Dirac comb can be applied to $C^{\infty}$ functions such that their product with the function $x \mapsto 1+x^{2}$ are bounded. 
The inequality (33) for $S_{+}$and the fact that $\psi$ is a bounded function hence imply that $\left\langle S_{+} D, \psi\right\rangle=\left\langle D, S_{+} \psi\right\rangle$ is well defined. Since we have $\chi_{[0, M-1]}(t) \leq S_{+}(t)$ for all $t \in \mathbb{R}$, we obtain that

$$
\sum_{m=0}^{M-1}|v(m)|^{2} \leq\left\langle D, S_{+}|v|^{2}\right\rangle .
$$

By using the fact that the Dirac comb is its own Fourier Transform, namely $D=\sum_{l \in \mathbb{Z}} e^{-2 \pi i l x}$, we obtain

$$
\sum_{m=0}^{M-1}|v(m)|^{2} \leq \sum_{l \in \mathbb{Z}}\left\langle e^{-2 \pi i l x}, S_{+}|v|^{2}\right\rangle
$$

and by using the definition of $v$,

$$
\sum_{m=0}^{M-1}|v(m)|^{2} \leq \sum_{n=0}^{N-1} \sum_{n^{\prime}=0}^{N-1} \sum_{l \in \mathbb{Z}} u_{n} \bar{u}_{n^{\prime}} \int_{\mathbb{R}} e^{-2 \pi i l x} S_{+}(x) e^{-2 \pi i\left(f_{n}-f_{n^{\prime}}\right) x} d x
$$

540

$$
=\sum_{n=0}^{N-1} \sum_{n^{\prime}=0}^{N-1} \sum_{l \in \mathbb{Z}} u_{n} \bar{u}_{n^{\prime}} \widehat{S}_{+}\left(f_{n}-f_{n^{\prime}}+l\right)=\widehat{S}_{+}(0) \sum_{n=0}^{N-1}\left|u_{n}\right|^{2} .
$$

The last equality is due to the fact that from the definition of $\Delta$ given by (21), for $n \neq n^{\prime}$ we have $1>\inf _{l \in \mathbb{Z}}\left|f_{n}-f_{n^{\prime}}+l\right| \geq \Delta$, while the support of $\widehat{S_{+}}$is contained in $[-\Delta, \Delta]$. Since

$$
\widehat{S}_{+}(0)=\int_{-\infty}^{+\infty} S_{+}(t) d t=M-1+1 / \Delta,
$$

we obtain

$$
\sum_{m=0}^{M-1}|v(m)|^{2} \leq(M-1+1 / \Delta)\|U\|^{2} .
$$

By proceeding similarly with the inequality $S_{-}(t) \leq \chi_{[0, M-1]}(t)$ for all $t \in \mathbb{R}$, we obtain that for all vector $U \in \mathbb{C}^{N}$

$$
(M-1-1 / \Delta)\|U\|^{2} \leq\|V U\|^{2} \leq(M-1+1 / \Delta)\|U\|^{2},
$$

which completes the proof.

\section{Appendix B: fundamental solution for a Dirac on the boundary}

Let us define $\mathcal{D}(\bar{W})$ as the set of restrictions to $W$ of functions in $\mathcal{D}\left(\mathbb{R}^{2}\right)$ and $L_{\text {loc }}^{2}(W)$ as the set of functions $u$ in $W$ such that $\phi u \in L^{2}(W)$ for all $\phi \in \mathcal{D}(\bar{W})$. 
For $y=\left(y_{1}, d\right)$, let us prove that the unique solution $G(\cdot, y)$ in $L_{\text {loc }}^{2}(W)$ of the problem

$$
\left\{\begin{array}{ccc}
\left(\Delta+k^{2}\right) G(\cdot, y)=0 & \text { in } & W \\
\partial_{\nu} G(\cdot, y)=\delta_{y_{1}} & \text { on } & \Gamma_{d} \\
\partial_{\nu} G(\cdot, y)=0 & \text { on } & \Gamma_{0} \\
(R C) & &
\end{array}\right.
$$

is given by

$$
G(x, y)=-\sum_{n \in \mathbb{N}} \frac{e^{i \beta_{n}\left|x_{1}-y_{1}\right|}}{2 i \beta_{n}} \theta_{n}\left(x_{2}\right) \theta_{n}(d) .
$$

We consider a solution $G \in L_{\text {loc }}^{2}(W)$ of problem 35 and a function $\phi \in \mathcal{D}(\bar{W})$ 555 such that $\partial_{\nu} \phi=0$ on $\Gamma$. Let us assume that the support of $\phi$ is contained in a rectangle $R=(-r, r) \times[0, d]$ and let $\Omega \subset W$ be an open smooth domain such that $\bar{R} \subset \bar{\Omega}$ and $\bar{R} \cap \bar{\Omega}=[-r, r] \times(\{0\} \cup\{d\})$. We observe that $G, \Delta G \in L_{\text {loc }}^{2}(W)$ hence

$$
\int_{W} \Delta G \phi d x+k^{2} \int_{W} G \phi d x=0 .
$$

Since $G, \Delta G \in L^{2}(\Omega)$ and $\phi \in H^{2}(\Omega)$, by an extended Green formula in the smooth domain $\Omega$ following [24, we have

$$
\int_{\Omega} \Delta G \phi d x=\int_{\Omega} G \Delta \phi d x+\left\langle\frac{\partial G}{\partial \nu}, \phi\right\rangle_{\partial \Omega}-\left\langle G, \frac{\partial \phi}{\partial \nu}\right\rangle_{\partial \Omega},
$$

where the first brackets mean duality between $H^{-3 / 2}(\partial \Omega)$ and $H^{3 / 2}(\partial \Omega)$ while the second brackets mean duality between $H^{-1 / 2}(\partial \Omega)$ and $H^{1 / 2}(\partial \Omega)$. The integrals on $\Omega$ above coincide with integrals on $W$. We hence obtain that

$$
-\int_{W} G\left(\Delta \phi+k^{2} \phi\right) d x=\left\langle\delta_{y}, \phi\right\rangle_{\Gamma_{d}}
$$

where the brackets mean duality between $H^{-3 / 2}\left(\Gamma_{d}\right)$ and $H^{3 / 2}\left(\Gamma_{d}\right)$. Let us ${ }_{565}$ choose $\phi=\phi_{n}$ with $\phi_{n}\left(x_{1}, x_{2}\right)=\psi\left(x_{1}\right) \theta_{n}\left(x_{2}\right)$ for any $\psi \in \mathcal{D}(\mathbb{R})$ the support of which is contained in $(-r, r)$. For all $r>0, G \in L^{2}((-r, r) \times(0, d))$. Since space $L^{2}((-r, r) \times(0, d))$ coincides with space $L^{2}\left((-r, r), L^{2}(0, d)\right)$ and the $\theta_{n}$ form a complete basis of $L^{2}(0, d)$, we can for almost all $x_{1}$ decompose $G(x)$ as

$$
G\left(x_{1}, x_{2}\right)=\sum_{n \in \mathbb{N}} a_{n}\left(x_{1}\right) \theta_{n}\left(x_{2}\right)
$$

and

$$
\|G\|_{L^{2}((-r, r) \times(0, d))}^{2}=\sum_{n \in \mathbb{N}}\left\|a_{n}\right\|_{L^{2}(-r, r)}^{2}<+\infty .
$$

In particular $a_{n} \in L_{\text {loc }}^{2}(\mathbb{R})$ for all $n$. By plugging these expressions of $G$ and $\phi$ in (37), and by using that $-\partial^{2} \theta_{n} / \partial x_{2}^{2}=\lambda_{n} \theta_{n}$ and $\beta_{n}^{2}=k^{2}-\lambda_{n}$, we end up with: for all $n \in \mathbb{N}$

$$
-\int_{\mathbb{R}} a_{n}\left(x_{1}\right)\left(\frac{\partial^{2} \psi}{\partial x_{1}^{2}}+\beta_{n}^{2} \psi\right) d x_{1}=\theta_{n}(d)\left\langle\delta_{y_{1}}, \psi\right\rangle, \quad \forall \psi \in \mathcal{D}(\mathbb{R}) .
$$


This exactly means that for all $n \in \mathbb{N}$, the function $a_{n}$ satisfies

$$
-\left(\frac{\partial^{2} a_{n}}{\partial x_{1}^{2}}+\beta_{n}^{2} a_{n}\right)=\theta_{n}(d) \delta_{y_{1}}
$$

in the distributional sense in $\mathbb{R}$. Since $\beta_{n} \neq 0$ for all $n$, a classical computation 575 enables us to conclude that

$$
a_{n}\left(x_{1}\right)=-\frac{\theta_{n}(d)}{2 i \beta_{n}} e^{i \beta_{n}\left|x_{1}-y_{1}\right|}+a_{n}^{+} e^{i \beta_{n} x_{1}}+a_{n}^{-} e^{-i \beta_{n} x_{1}} .
$$

The radiation condition implies that $a_{n}^{+}=0$ and $a_{n}^{-}=0$ for all $n$ and we obtain the desired expression of $G(\cdot, y)$ given by (36). Conversely, for $G(\cdot, y)$ given by (36), $G(\cdot, y)$ satisfies the problem (35). It remains to check that $G(\cdot, y) \in$ $L_{\text {loc }}^{2}(W)$, which is a straightforward consequence of the expression of the $a_{n}$ in 580 view of 38 .

\section{Acknowledgements}

The authors are indebted to Alexandre Impériale, who helped them to use the time domain code ECHO developed by himself at the CEA. They also thank Anne-Claire Egloffe and Eric Lunéville, who initiated this work with the second author during the Post-doc of Anne-Claire Egloffe at ENSTA ParisTech.

\section{References}

[1] L. Bourgeois, E. Lunéville, The linear sampling method in a waveguide: a modal formulation, Inverse Problems 24 (1) (2008) 015018. doi:10.1088/ 0266-5611/24/1/015018.

${ }_{590}$ URL http://dx.doi.org/10.1088/0266-5611/24/1/015018

[2] T. Druet, B. Chapuis, M. Jules, G. Laffont, E. Moulin, Pzt array for passive guided wave tomography of extended defects using ambient elastic noise cross-correlations, 7th Int. Symposium on NDT in Aerospace (2015) Tu.2.A.4.

595

[3] P. Huthwaite, Evaluation of inversion approaches for guided wave thickness mapping, Proceedings of the Royal Society of London A: Mathematical,

11 Physical and Engineering Sciences 470 (2166). arXiv:http://rspa. royalsocietypublishing.org/content/470/2166/20140063.full.pdf, doi:10.1098/rspa.2014.0063.

URL http://rspa.royalsocietypublishing.org/content/470/2166/ 20140063

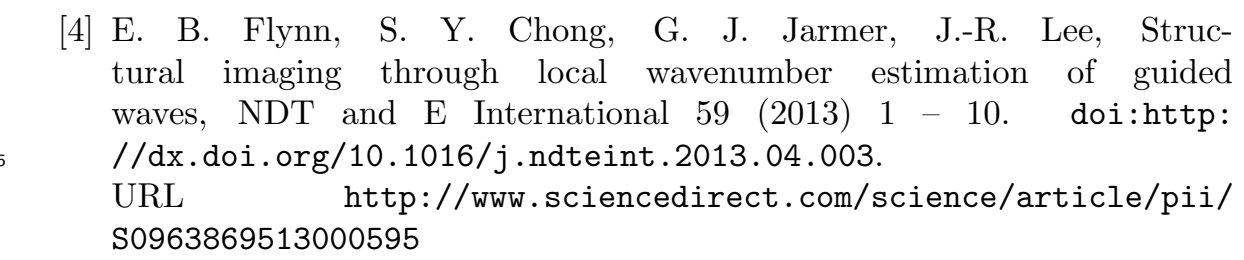


[5] J.-L. Lions, E. Magenes, Problèmes aux limites non homogènes et applications. Vol. 2, Travaux et Recherches Mathématiques, No. 18, Dunod, Paris, 1968.

[6] D. Colton, A. Kirsch, A simple method for solving inverse scattering problems in the resonance region, Inverse Problems 12 (1996) 383-393.

[7] F. Cakoni, D. Colton, Qualitative methods in inverse scattering theory, Interaction of Mechanics and Mathematics, Springer-Verlag, Berlin, 2006.

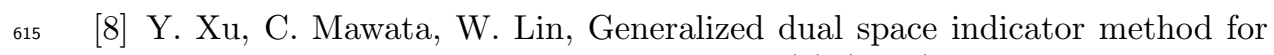
underwater imaging, Inverse Problems 16 (6) (2000) 1761-1776. URL http://stacks .iop.org/0266-5611/16/i=6/a=311

[9] A. Charalambopoulos, D. Gintides, K. Kiriaki, A. Kirsch, The factorization method for an acoustic wave guide, 7th Int. Workshop on Mathematical Methods in Scattering Theory and Biomedical Engineering (2006) 120-127.

[10] P. Monk, V. Selgas, Sampling type methods for an inverse waveguide problem, Inverse Problems and Imaging 6 (4) (2012) 709-747.

[11] L. Bourgeois, E. Lunéville, On the use of sampling methods to identify cracks in acoustic waveguides, Inverse Problems 28 (10) (2012) 105011. doi: $10.1088 / 0266-5611 / 28 / 10 / 105011$. URL http://dx.doi .org/10.1088/0266-5611/28/10/105011

[12] L. Bourgeois, F. Le Louer, E. Lunéville, On the use of lamb modes in the linear sampling method for elastic waveguides, Inverse Problems 27 (5) (2011) 055001. URL http://stacks .iop.org/0266-5611/27/i=5/a=055001

[13] L. Bourgeois, E. Lunéville, On the use of the linear sampling method to identify cracks in elastic waveguides, Inverse Problems 29 (2) (2013) 025017. URL http://stacks .iop.org/0266-5611/29/i=2/a=025017

[14] Q. Chen, H. Haddar, A. Lechleiter, P. Monk, A sampling method for inverse scattering in the time domain, Inverse Problems 26 (8) (2010) 085001, 17. doi:10.1088/0266-5611/26/8/085001. URL http://dx.doi.org/10.1088/0266-5611/26/8/085001

[15] P. Monk, V. Selgas, An inverse acoustic waveguide problem in the time domain, Inverse Problems 32 (5) (2016) 055001. URL http://stacks .iop.org/0266-5611/32/i=5/a=055001

[16] S. Fliss, P. Joly, Solutions of the time-harmonic wave equation in periodic waveguides: Asymptotic behaviour and radiation condition, Archive for

q Rational Mechanics and Analysis 219 (1) (2015) 349-386. doi:10.1007/ s00205-015-0897-3. URL http://dx.doi.org/10.1007/s00205-015-0897-3 
[17] L. Bourgeois, E. Lunéville, The linear sampling method in a waveguide: a formulation based on modes, Journal of Physics: Conference Series 135 (2008) 012023.

[18] A. Moitra, Super-resolution, extremal functions and the condition number of Vandermonde matrices, in: STOC'15-Proceedings of the 2015 ACM Symposium on Theory of Computing, ACM, New York, 2015, pp. 821-830.

[19] M. Cassier, C. Hazard, Space-time focusing of acoustic waves on unknown scatterers, Wave Motion 51 (8) (2014) 1254-1272. doi:10.1016/j. wavemoti.2014.07.009. URL http://dx.doi.org/10.1016/j.wavemoti.2014.07.009

[20] B. B. Guzina, F. Cakoni, C. Bellis, On the multi-frequency obstacle reconstruction via the linear sampling method, Inverse Problems 26 (12) (2010) 125005, 29. doi:10.1088/0266-5611/26/12/125005. URL http://dx.doi.org/10.1088/0266-5611/26/12/125005

[21] J. D. D. Basabe, M. K. Sen, Grid dispersion and stability criteria of some common finite-element methods for acoustic and elastic wave equations,

1. GEOPHYSICS 72 (6) (2007) T81-T95. arXiv:http://dx.doi.org/10. 1190/1.2785046, doi:10.1190/1.2785046 URL http://dx.doi.org/10.1190/1.2785046

[22] P. Joly, An elementary introduction to the construction and the analysis of perfectly matched layers for time domain wave propagation, SëMA J. (57) (2012) 5-48.

[23] H. L. Montgomery, Harmonic analysis as found in analytic number theory, in: Twentieth century harmonic analysis - a celebration (Il Ciocco, 2000), Vol. 33 of NATO Sci. Ser. II Math. Phys. Chem., Kluwer Acad. Publ., Dordrecht, 2001, pp. 271-293.

[24] J.-L. Lions, E. Magenes, Problèmes aux limites non homogènes et applications. Vol. 1, Travaux et Recherches Mathématiques, No. 17, Dunod, Paris, 1968 . 